\title{
UCRL 8621
}
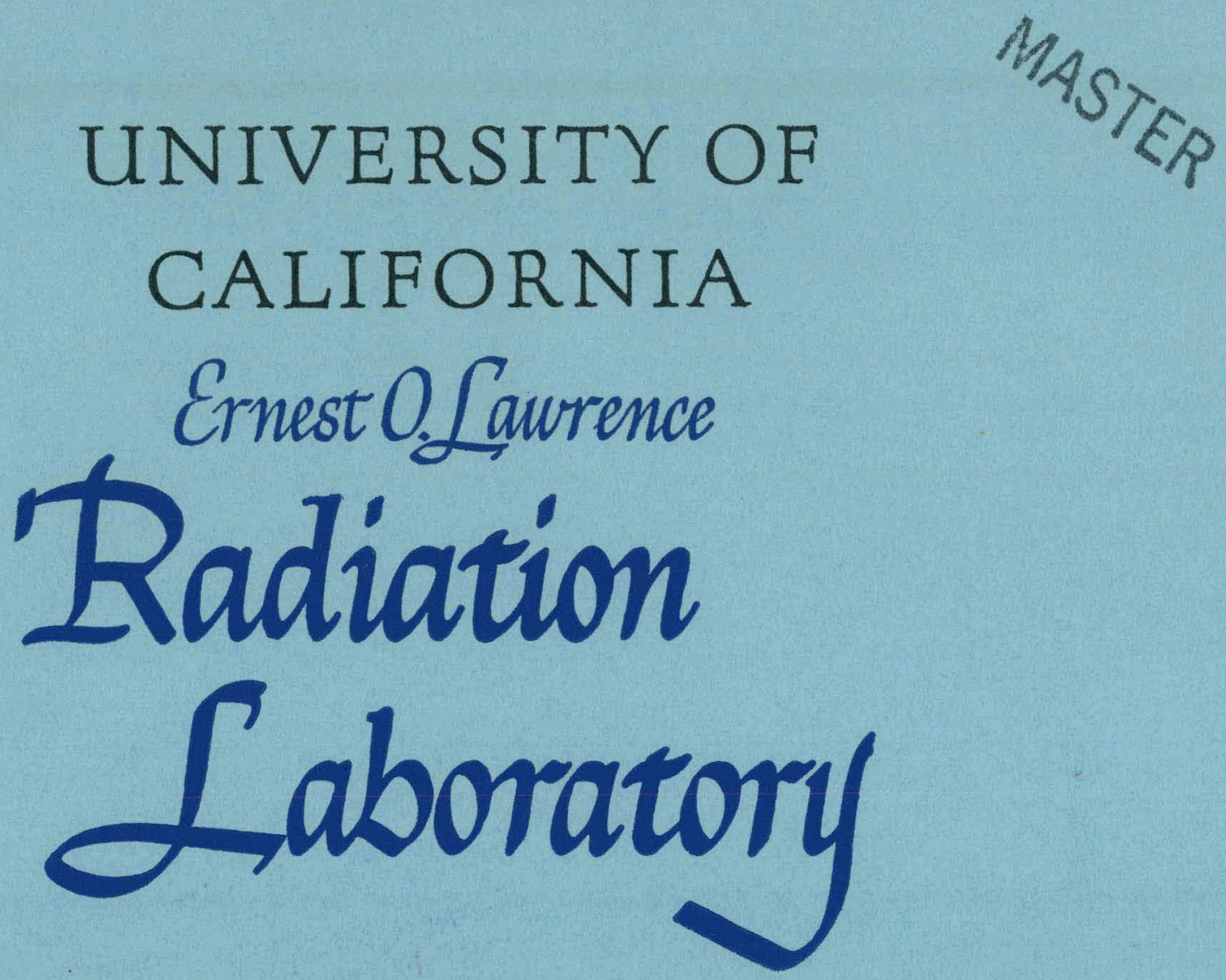

A PROPOSAL FOR DETERMINING

THE ELECTROMAGNETIC FORM FACTOR OF THE PION

\author{
BERKELEY, CALIFORNIA
}




\section{DISCLAIMER}

This report was prepared as an account of work sponsored by an agency of the United States Government. Neither the United States Government nor any agency Thereof, nor any of their employees, makes any warranty, express or implied, or assumes any legal liability or responsibility for the accuracy, completeness, or usefulness of any information, apparatus, product, or process disclosed, or represents that its use would not infringe privately owned rights. Reference herein to any specific commercial product, process, or service by trade name, trademark, manufacturer, or otherwise does not necessarily constitute or imply its endorsement, recommendation, or favoring by the United States Government or any agency thereof. The views and opinions of authors expressed herein do not necessarily state or reflect those of the United States Government or any agency thereof. 


\section{DISCLAIMER}

Portions of this document may be illegible in electronic image products. Images are produced from the best available original document. 
UNIVERSITY OF CALIFORNIA

\section{Lawrence Radiation Laboratory \\ Berkeley, California \\ Contract No. W-7405-eng-48}

A PROPOSAL FOR DETERMINING

THE ELECTRUMAGNETIC FURM FACTOR OF THE PION

$$
\begin{gathered}
\text { William Robert Frazer } \\
\text { (Thesis) }
\end{gathered}
$$

February 4, 1959

Printed for the U. S: Atomic Energy:Commission 
A PROPOSAL FOR DETERMINING

THE ELECTROMAGNETIC FORM FACTOR. OF THE PION

Contents

Abstract .......................... 3

I. Introduction .................... 4 II. Extrapolation Procedure

A. Location of Singularities of Scattering Amplitude . . 8

B. The Extrapolation Formula. . . ........ 10

C. Kinematical Considerations ............ 13

III. Estimate of Electroproduction Cross Section

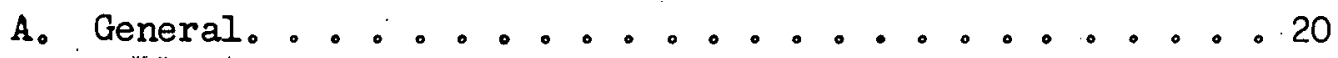

B. Dispersion Theory of Photoproduction and Electroproduction. . . . . . . . . . . . . 23

C. Calculation of the Photoproduction Cross Section . . . 36:

D. Calculation of the Electroproduction Cross Section . . 39

IV. Conclusions .................... 44 Acknowledgments. . . . . . . . . . ...... 45 Figure Legends ...................... 46

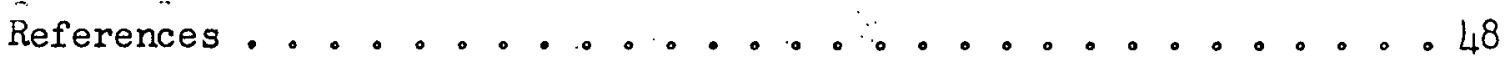


A PROPOSAL FOR DETERMINING

THE ELECTROMAGNETIC FORM FACTOR OF THE PION

William Robert Frazer

(Thesis)

Lawrence Radiation Laboratory
University of California
Berkeley, California

February 4, 1959

ABSTRACT

The possibility of measuring the electromagnetic form factor of the pion by extrapolation of the cross section for $e^{-}+p \rightarrow n+\pi^{+}+e^{-}$ has been investigated. The method is based on the existence of a pole in the electropion-production scattering amplitude as a function of the invariant momentum-transfer of the nucleon. The residue of this pole is the pion form factor multiplied by a known coefficient. Since the pole lies slightly outside the physical region of the invariant momentum transfer, an extrapolation of the experimental data is required. An approximate calculation of the electropion-production cross section has been made in order to estimate the experimental accuracy necessary for a significant extrapolation. Accuracy is required which is an order of magnitude better than that achieved at present in similar experiments. 


\section{INTRODUCTION}

In recent years much attention has been devoted to the problem of the electromagnetic structure of the nucleon. Considerable experimental information on this subject has been provided by the experiments carried out by Hofstadter and his collaborators on the scattering of electrons by protons and deuterons. ${ }^{1}$ Additional information was derived from the measurement of the cross section for electropion production $\left(e^{-}+p \rightarrow e^{-}+n+\pi^{+}\right)$ by Panofsky and Allton.?

Among the many theoretical attempts which have been made to treat the nucleon-structure problem, the most successful have been the two recent calculations based on the method of spectral representations (often called dispersion relations) 3,4 In this method, however, one encounters the difficulty of requiring knowledge of the electromagnetic structure of the pion. It is qualitatively evident that the structure of the pion must contribute to the structure of the nucleon: If the spatial extension of the nucleon's charge and magnetic moment are visualized as due to the pion cloud of the nucleon, then the spatial extension of the pion will contribute to that of the nucleon.

Unfortunately, no experiments have been performed which probe the pion structure. The scattering of pions on electrons yields no information at available energies, because the relatively massive incident pion cannot transfer sufficient momentum to make anything but the outermost parts of the pion electromagnetic field effective. On the other hand, scattering of electrons on pions is not feasible because, of course, no way has been found for making targets from pions. (The purpose of this thesis is to describe a method of using the pion cloud of the proton as a "pion target." 
The procedure to be described is an application of a general method suggested recently by Chew and Low. 5 In this case, their method enables. one to measure the electromagnetic form factor of the pion by extrapolation of the cross section for $\mathrm{e}^{-}+\mathrm{p} \rightarrow \mathrm{n}+\dot{\pi}^{+}+\mathrm{e}^{-}$. The basic principle involved is the possibility of analytic continuation of the electroproduction scattering amplitude as a function of the square of the four-momentum transfer of the nucleon, $\Delta^{2}$. If the initial nucleon has four-momentum $p$, and the final nucleon, $p^{\prime}$, then we have $\Delta^{2}=-\left(p-p^{p}\right)^{2}$. * It is conjectured that at $\Delta^{2}=-\mu^{2}$, the scattering amplitude has an isolated pole whose residue is just the electromagnetic form factor of the pion multiplied by a known coefficient. It can easily be shown that negative values of $\Delta^{2}$ are not physically attainable, so that an extrapolation of the measured cross section is necessary in order to reach the pole at $\Delta^{2}=-\mu^{2}$. The distance of extrapolation is, however, small compared to the physical range of $\Delta^{2}$. The procedure for determining the electromagnetic form factor of the pion is, then, to extrapolate the cross section, with its singularities suitably removed, to the point $\Delta^{2}=-\mu^{2}$. This procedure will be described in detail in Section II.

Experimentally, it will be necessary to measure the electroproduction: cross section as a function of at least two variables, $\Delta^{2}$ and $\lambda$. If $s$ is the incident electron four-momentum, and $s^{\prime}$ is the final, then we have $\lambda=-\left(s-s^{\prime}\right)^{2}$. One must know $\lambda$ because the pion form factor is a function of this variable. In practice, one could determine these variables

The metric tensor $g^{00}=1, g^{11}=-1$ for $i=1,2$, or 3 is used throughout. Units are used in which $\not h=c=1$, and usually $\mu=1$, where $\mu$ is the mass of the pion. 
by measuring the energy and direction of the final electron, plus the direction of the final meson. No such measurements have yet been made. In their recent experiment, Panofsky and Allton ${ }^{2}$ measuréd the electron variables but did not detect the meson directly......

The analytic properties of the electroproduction scattering amplitude, which are the basis of the method outlined above, have not been proved rigorously. They are, however, a very reasonable extension of properties which have been proved for other scattering problems. A plausibility argument will be given in Section II A. Such analytic properties of scattering amplitudes have been the object of much study. Their application in the form of spectral representations has been successful in correlating many experimental data in pion physics. Applications very similar to that proposed in this thesis have already been made for the purpose of measuring the pion-nucleon coupling constant. Extrapolations of both the nucleon-nucleon ${ }^{6,7}$ and photopion-production ${ }^{8}$ cross sections as functions of invariant momentum transfer yielded values of the coupling constant in reasonable agreement with values obtained by other means.

In principle, then, the analytic properties of the electroproduction scattering amplitude described above tell us that one can determine the pion form factor by an extrapolation procedure. In order to assess the practical difficulty involved in performing an extrapolation of a given set of experimental data, one must estimate the behavior of the electroproduction cross section as a function of $\Delta^{2}$. If the electromagnetic interaction is treated in lowest order of perturbation theory, it is apparent that the electroproduction and photoproduction matrix elements are closely related. The dispersion theoretical analysis of photoproduction by Chew, 
Goldberger, Iow, and Nambu ${ }^{9}$ (hereafter called CGLN) has been extended to electroproduction by Fubini, Nambu, and Wataghin ${ }^{10}$ (hereafter FNW). The calculation in section III is performed by the use of a modification of theix theory: The Born terms in the matrix element are written in relativistic form and, correspondingly, certain recoil-correction terms are dropped.

In Section IV the results of this calculation are interpreted as implying that for a significant extrapolation experiments of great accuracy will be necessary, accuracy an order of magnitude better than that achieved in the electroproduction experiment of Panofsky and Allton.? 


\section{EXTIRAPOLATION PROCEDURE}

\section{A. Location of Singularities of Scattering Amplitude.}

Let us consider in detail the analytic properties of the electropionproduction scattering amplitude on which the proposed extrapolation depends. As remarked in the introduction, no rigorous proof of these properties has been given. However, such properties have often been conjectured; for instance, in the two-dimensional spectral representation proposed by Mandelstam ${ }^{11}$ and verified to sixth order in perturbation theory. Motivation for conjecturing them comes from two sources: analogy with proved properties of simpler scattering amplitudes and analysis of perturbation theory. Arguments of both types have been given by chew ${ }^{6}$ for nucleon-nucleon scattering, and by Taylor, Moravcsik, and Uretsky ${ }^{8}$ (hereafter TMU) for photoproduction. The latter case can be extended very easily to electroproduction. Consider the electron interaction only in lowest order in the fine structure constant, i.e., consider only processes of the type shown in Fig. 1. To this order, electroproduction is just photoproduction by a virtual photon. The only differences are that the , "photon" has a nonzero, imaginary mass $\left(\mathrm{k}^{2}=-\lambda\right)$ in electroproduction, and that the matrix element contains longitudinal as well as transverse terms (i.e., $\overrightarrow{\mathrm{k}} \cdot \vec{\epsilon} \neq 0$ ). The existence of transverse terms will not affect the analytic properties, and the "photon" mass will only cause a shift in the position of the singularities.

The recipe used in the papers referred to above is the following: To get one part of the spectrum consider the intermediate states which could be reached if $p$ and $p^{\prime}$ were incoming particles, with $q$ and $\mathrm{k}$ outgoing ( 
discrete single pion state, which gives a pole at $\Delta^{2}=-\mu^{2}$ (on which the proposed extrapolation is based). The next state is that of two pions, which gives rise to a branch point at $\Delta^{2}=-4 \mu^{2}$.

The other half of the spectrum, the crossed spectrum, is found in a similar way by considering the states which can be reached if $p$ and $q$ are incident. This leads to a pole at $(p-q)^{2}=M^{2}$, where $M$ is the nucleon mass, and a branch point at $\cdot(p-q)^{2}=(M+\mu)^{2}$. Now since $p+k=p^{\prime}+q$, we find that

$$
(p-q)^{2}=\Delta^{2}-E^{2}+2 M^{2}+\mu^{2}-\lambda .
$$

Here $E^{2}=\left(p^{\prime}+q\right)^{2}$; i.e., $E$ is the total energy of the final nucleon and pion in their barycentric system (the system in which $\left.\vec{p}^{\prime}+\vec{q}=0\right)$. Using $(2.1)$, one finds that the crossed spectrum gives rise to a pole at

$$
\Delta^{2}=E^{2}-M^{2}-\mu^{2}+\lambda,
$$

and a branch point at

$$
\Delta^{2}=E^{2} \cdot M^{2}+2 M \mu+\lambda \cdot \cdot
$$

The spectrum of singularities in the $\Delta^{2}$ plane can be re-expressed in terms of $\cos \theta$, where $\theta$ is the angle between $\vec{q}$ and $\vec{k}$ in the $\vec{p}^{\prime}+\vec{q}=0$ system. With all symbols referring to this system, we have

$$
\Delta^{2}=\lambda-\mu^{2}+2 \omega_{2} k_{0}-2|\vec{q}||\vec{k}| \cos \theta
$$


where

$$
\omega_{2} \equiv \sqrt{|\vec{q}|^{2}+\mu^{2}}
$$

Then in the $\cos \theta$ plane the analyticity region is the cut plane, with poles and branch points as shown in Fig. 2. The quantity $\mathrm{E}_{2}$ is the energy of the final nucleon. In the limit $\lambda=0$ the positions of the singularities reduce to those of photoproduction given by TMU. Actually, the existence of such a large region of analyticity is not necessary for the proposed extrapolation. The method requires only that we have analyticity in some region containing the physical region $|\cos \theta| \leq I$ and including the pole at $\cos \theta=\left(\lambda+2 a \mathrm{k}_{0}\right) / 2|\overrightarrow{\mathrm{q}}||\overrightarrow{\mathrm{k}}|$ as an isolated singularity。

\section{B. The Extrapolation Formula。}

If one accepts the viewpoint that perturbation theory can yield information on analytic behavior, then the existence of the pole at $\Delta^{2}=-\mu^{2}$ can be demonstrated and its residue computed. It is evident that the class of diagrams shown in Fig. 3 gives rise to the pole. It is easy to show that no other type of diagram can contain this pole. The contribution of this diagram ${ }^{*}$ to the cross section gives, in covariant form,

$\frac{d \sigma}{d P}=\frac{4 g^{2} \alpha^{2} \Delta^{2} F_{\pi}^{2}(\lambda)}{\lambda^{2} \cdot \pi^{2} I\left(\Delta^{2}+\mu^{2}\right)^{2}}\left[4(q \cdot s)\left(q \cdot s^{0}\right)-\mu^{2} \lambda\right]+$ other terms,

The notation used for the coupling constants (renormalized) is such that $g^{2}=\frac{g_{r}}{4 \pi}, f=\frac{\mu}{2 M} g$, and $f^{2} \approx 0.08$. 
where $I \equiv \sqrt{(p \cdot s)^{2}-M^{2} m^{2}}=M s_{L}$, and where $d P$ is the phase space factor:

$\mathrm{dP} \equiv \frac{\mathrm{a}^{3} \mathrm{p}^{3} \mathrm{~d}^{3} \mathrm{~s}^{3} \mathrm{~d}^{3} \mathrm{q}}{8 \omega_{2} \mathrm{E}_{2} \varepsilon_{2 . ;}} \delta^{4}\left(p+s-p^{\prime}-s^{\prime}-q\right)$

The symbols $\epsilon_{1}, \epsilon_{2}$ are defined as the initial and final electron energies. One finds that

$d P=\frac{\pi|\vec{q}|^{2} d \lambda d E^{2} d \Omega_{q} .}{16 M s_{L} \omega_{2}\left|\frac{d}{d|\vec{q}|} q \cdot\left(p+s-s^{0}\right)\right|}$,

where $d s_{q}$ refers to the outgoing meson. Since $d P$ as defined by Eq. (2.4) is a Lorentz invariant, this expression must be valid in any coordinate system. In the lab system, we have

$d P_{I}=\frac{\pi|\vec{q}|^{2} d \lambda d E^{2} d \Omega_{q}}{16 M s_{L}\left[|\vec{q}|\left(M+k_{0}\right)-\omega_{2}|\vec{k}| \cos \theta\right]}$.

In Eq. (2.3) two quantities in the numerator, $g$ and $F_{\pi}(\lambda)$, are not the most general expressions corresponding to the diagram of Fig. 3. They have been given the value appropriate to $\Delta^{2}=-\mu^{2}$ in anticipation of the extrapolation to that point. The pion form factor, $F_{\pi}(\lambda)$, is defined by considering the pion-photon vertex, Fig. 4, with both pions on the mass shell. If we write the contribution of this vertex as $j \mu\left(q_{1} ; q_{2}\right) \epsilon^{\mu}$, the most general form of $j$ consistent with Lorentz invariance is 


$$
j\left(q_{1}, q_{2}\right)=-e F_{\pi}(\lambda) q_{I}-e G_{\pi}(\lambda) q_{2}
$$

where $\lambda=-\left(q_{2}-q_{1}\right)^{2}$. The continuity equation imposes the further requirement $\left(q_{2}-q_{1}\right) \cdot j=0$, giving $F_{\pi}(\lambda)=G_{\pi}(\lambda)$, or

$$
j\left(q_{1}, q_{2}\right)=-\left(q_{1}+q_{2}\right) e F_{\pi}(\lambda)
$$

This definition of the pion form factor is normalized so that $F_{\pi}(0)=1$. Equation (2.3) reveals an additional singularity which must be removed before an extrapolation can be performed. The factor in brackets, which results from taking the trace of matrix factors, has the form, as a function of $\cos \theta: P_{1}(\cos \theta)+\sin \theta P_{2}(\cos \theta)$, where $P_{1}$ and $P_{2}$ are polynomials. Now since $\sin \theta=\left(1-\cos ^{2} \theta\right)^{1 / 2}, \sin \theta$ has a branch point at $\cos \theta=1$, preventing extrapolation. We cannot get rid of this singularity by division by the factor. in brackets, since it can vanish in the region of extrapolation and does vanish in the case to be discussed in Section III. We can, however, eliminate this undesirable $\sin \theta$ by defining a "symmetrized" cross section. Define $\sigma(\theta) \equiv \mathrm{d} \sigma / \mathrm{dP}$ 。The quantity $\sigma(\theta)$. does, of course, depend upon variables other than $\theta$ 。 Then define the symmetrized quantity ${ }^{*}$.

$$
\langle\sigma(\cos \theta)\rangle_{s} \equiv \sigma(\theta)+\sigma(-\theta) .
$$

The quantity $\langle\sigma(\cos \theta)\rangle_{s}$ will, of course, be free from the branch point

*

See Eq. (3.48) for an equivalent definition of this symmetrization process. 
at $\cos \theta=1$, and we can at last write the extrapolation formula:

$$
\left.\left(\Delta^{2}+\mu^{2}\right)^{2}\langle\sigma\rangle s\right|_{\Delta^{2}=-\mu}{ }^{2}=-\left.\frac{4 g^{2} \alpha^{2} \mu^{2} F_{\pi}^{2}(\lambda)}{\pi^{2} \cdot M s_{L} \lambda^{2}}\left\langle 4(q \cdot s)\left(q \cdot s^{0}\right)-\mu^{2} \lambda\right\rangle_{s}\right|_{\Delta^{2}=-\mu^{2}}
$$

The above discussion has shown that if one knew the value of the differential cross section $\mathrm{d} \sigma / \mathrm{dP}$ over some portion of the physical region, one could obtain the value of $F_{\pi}(\lambda)$ by analytic continuation of the function $\left(\Delta^{2}+\mu^{2}\right)^{2}\langle\sigma(\cos \theta)\rangle_{s}$. The relation between the value of this function at $\Delta^{2}=-\mu^{2}$, and the pion form factor is given by $\mathrm{Eq} \cdot(2.8)$. This is an idealization, of course, since in practice one can know the function only to within a certain error and at a finite number of points. One practical procedure that can be employed is to plot the experimental value of $\left(\Delta^{2}+\mu^{2}\right)^{2}\langle\sigma\rangle_{s}$ and fit a polynomial in $\cos \theta$ to these points by the method of least squares. ${ }^{2,12}$ The residue is then given by the value of this polynomial at the pole. Some consideration will be given in the next section to the error involved in this method of extrapolation.

\section{Kinematical Considerations.}

In order to perform the proposed extrapolation, it is necessary to know the cross section as a function of both the extrapolation variable $\Delta^{2}$ and the variable $\lambda$ on which the form factor $F_{\pi}(\lambda)$ depenas. Since an N-particle (incoming plus outgoing) scattering problem is a function of $3 \mathrm{~N}$ - 10 variables (neglecting spins), electroproduction is a function of five variables. A convenient choice for the other three variables 
is $E$, the total energy of the pion and nucleon in the $\vec{p}^{\prime}+\vec{q}=0$ system; $T_{L}$, the laboratory kinetic energy of the incident electron; and $\varnothing$, defined in the $\vec{p}^{\cdot}+\vec{q}=0$ system by

$$
\cos \varnothing=\frac{(\vec{k} \times \vec{s}) \cdot(\vec{k} \times \vec{q})}{|\vec{k} \times \vec{s}||\vec{k} \times \vec{q}|}
$$

In principle these three variables could be integrated out and the cross section measured as a function of $\Delta^{2}$ and $\lambda$. only. In practice, it may be most convenient to determine all five variables; for instance, by knowing the incident-electron energy and measuring the distribution of mesons as a function of direction, in coincidence with final electrons of given direction and energy. Then in performing the extrapolation all variables except $\Delta^{2}$ must be held fixed. The question then arises: what values of $\dot{E}, \mathrm{~T}_{\mathrm{I}}$, and $\varnothing$ are most favorable to the extrapolation procedure?

To answer this question we must be able to estimate the error associated with extrapolation. Let us assume that the extrapolation will be done by fitting a polynomial in $\cos \theta$ to the function $\left(\Delta^{2}+\mu^{2}\right)^{2}\langle\sigma\rangle s$ by the method of least squares. Then we can use a well-known formula ${ }^{12}$ to calculate the error.

Let $x_{0}$ be the position of the pole as a function of $\cos \theta$. Then define

$$
\mathrm{x} \equiv \mathrm{x}_{0}-\cos \theta
$$

In the $\vec{p}^{0}+\vec{q}=0$ system, we find

$$
\Delta^{2}+\mu^{2}=2|\vec{q}||\vec{k}| x \text {. }
$$


We. wish to extrapolate the function

$$
f(x) \equiv(2|\vec{q}||\vec{k} \cdot|)^{2} x^{2} \cdot\langle\sigma\rangle_{s}
$$

to the point $x=0$, the position of the pole of $\langle\sigma\rangle_{s}{ }^{\circ}$ The error in the leastisquares polynomial at the point $x=0$ is given by

$$
\Delta_{0}=\rho \sqrt{\left(h^{-1}\right)_{00}}
$$

where

$$
\mathrm{h}_{\mathrm{mn}} \equiv \sum_{i=1}^{\mathrm{N}} \frac{\mathrm{x}_{i}^{\mathrm{m}+\mathrm{n}}}{\zeta_{i}^{2}}
$$

and where $\rho$, which depends upon the goodness of fit, is $\approx 1.12$ The summation in Eq. (2.11') extends over the points at which $f(x)$ is known, and $\zeta_{i}$ is the experimentally determined standard deviation of $f(x)$ at $x_{i}$. In order to use Eq. (2.11) to determine the dependence of $\triangle a_{0}$ on the experimental parameters, we must make some assumption about the behavior of the $\zeta_{i}{ }^{\prime} s$. Let us assume that there is a constant standard deviation $\xi$ in the cross section $\langle\sigma\rangle_{s}$ for all values of $x$. This seems to be the most reasonable assumption to make in the absence of detailed knowledge of the behavior of $\langle\sigma\rangle_{S}{ }^{\circ}$ This assumption implies that the standard deviation $\zeta_{i}$ of $f(x)$ is

$$
\zeta_{i}=(2|\vec{q}||\vec{k}|)^{2} x_{i}^{2} \xi
$$

Then if $\rho=1, \mathrm{Eq} \cdot(2.11)$ becomes

$$
\Delta a_{0}=\xi(2|\vec{q}||\vec{k}|)^{2} \sqrt{\left(H^{-1}\right)_{0 O}} \text {, }
$$


where

$$
H_{m n} \equiv \sum_{i=1}^{N} x_{i}^{m+n-4} 。
$$

Notice that the error $\Delta_{0}$ is proportional to the absolute error in the measured cross section, and to the factor $\sqrt{\left(\mathrm{H}^{-1}\right)_{0 O}}$ which depends only. upon the distance of extrapolation and the distribution of points in the physical region.

It is qualitatively evident that the error will rise with the distance of extrapolation. Let us calculate the distance of extrapolation as a function of $E$ and $\lambda$. Equation (2.2) shows thst the pole occurs at

$$
\cos \theta=\left(\lambda+2 \omega_{2} k_{0}|/ 2| \vec{q}|| \vec{k} \mid \equiv x_{0}\right.
$$

Holding $\lambda$ fixed, one finds that all quantities on the right-hand side are known functions of $E$. One can easily show that in the $\vec{p}^{r}+\vec{q}=0$ system the following relations hold:

$$
\begin{aligned}
& 2 \omega_{2}=E-\left(M^{2}-\mu^{2}\right) / E \\
& 2 k_{0}=E-\left(M^{2}+\lambda\right) / E \\
& E_{2}=E-\omega_{2}=\sqrt{|\vec{q}|^{2}+M^{2}} \\
& E_{1}=E-k_{0}=\sqrt{|\vec{k}|^{2}+M^{2}} .
\end{aligned}
$$

The distance of extrapolation as a function of $E$, for two values of $\lambda$ is. shown in Fig. 5 .

In order to evaluate the dependence of $\Delta_{0}$ on the distance of extrapolation, we must calculate $\left(\mathrm{H}^{-1}\right)_{00^{\circ}}$. If we assume that we can fit $f(x)$ with a polynomial of the fourth order ( $S$ - and $P$-waves only); 
then $\mathrm{H}_{\mathrm{mn}}$ is a five-by-five matrix. The inversion of this matrix was performed by a machine calculation for various distances of extrapolation for the arbitrary case of eleven points $x_{i}$ spread evenly over the physical region. The results are summarized in Fig. $5 a$, where. $\Delta a$ g $/ \xi$ is shown as a function of $E$, for $\lambda=10$. If $\xi$ does not vary much with $E$, higher values of $E$ are clearly favored by this consideration.

A second consideration is the size of the residue at the pole. For a given $\Delta a_{0}$, the size of the residue determines the per cent error. From Eq. (2.8) one sees that for a given value of $\lambda$ and $T_{L}$ the residue is proportional to

$$
\left\langle 4(q \cdot s)\left(q \cdot s^{\prime}\right)-\mu^{2} \lambda\right\rangle_{s}
$$

In Fig. 6 this factor is plotted against $E$ for two extreme values of $\varnothing$. If one chooses $\phi=0$ or $\pi$, the size of the residue decreases with $E$. This decrease is, however, greatly outweighted by the more rapid decrease of $\Delta a_{0} / \xi$ with $E$ shown in Fig. 5a. The conclusion indicated is that unless experimental conditions create very strong variations with $\mathrm{E}$ in the absolute accuracy attainable; high values of $E$ are desirable. On the other hand, if $E$ is too high, one will be forced to use a polynomial of higher than fourth order to fit $f(x)$; i.e., D-waves will become important. The machine calculation of $\left(\mathrm{H}^{-1}\right)_{0 O}$. showed that the error increases markedly with the order of the polynomial. The error was calculated in the same manner as for Fig. 5 a for the point $E=9.66$ for fifth- and sixth-order polynomials. For the fourth-order polynomial, Fig. 5a shows $\Delta a_{0} / \xi=134$. For the fifth-order, we found $\Delta_{0} / \xi=345$, and for the sixth-order, $\Delta_{0} / \xi=952$. 
By machine calculation of the error for several specific distributions of points $\mathrm{x}_{1}$, some qualitative conclusions were drawn concerning the most favorable distribution. First, it seems desirable to have measurements spread over as wide a region as possible, preferably the entire range of. $\cos \theta$. Second, it seems desirable to concentrate most of the points close to $\cos \theta=1$. For example, at $E=9.66$ we saw that for eleven evenly spaced points $\Delta a_{j} / \xi=134$. For nine points at $\cos \theta=1,0.98,0.94$, $0.87,0.71,0.26,0,-0.71$, and -1 , we found $\Delta a_{0} / \xi=95$. Third, it is of course desirable to have as many points as possible. For 6, 11, 14, and 21 evenly spaced points $(E=9.66$, fourth-order polynomial $) ; \Delta_{0} / \xi=423$, 134, 100 and 75 , respectively.

It is beyond the scope of this work to provide a definitive answer to the experimental question of the optimum values of the parameters for the purpose of the extrapolation. An attempt has been made in this section to discuss the most important factors. In Section IIID a calculation will be made of the electroproduction cross section in order to translate $\mathrm{Eq}_{0}(2.12)$ into an estimate of the maximum per cent error compatible with a significant extrapolation. It is desirable, of course, to carry out this calculation at the most favorable value of the parameters. In order to consider a more familiar parameter, let us introduce the equivalent photoproduction energy $\dot{\mathrm{k}}_{\mathrm{ph}}$, defined by

$$
k_{p h}=\frac{E^{2}-M^{2}}{2 M} \text {. }
$$

The quantity $\mathrm{k}_{\mathrm{ph}}$ is the energy a photon must have in the laboratory in order to produce a pion-nucleon final state having total energy $E$ in the barycentric system. On the basis of the considerations discussed 
in this section, a reasonable guess for the most promising value of $\mathrm{k} p$ is $\approx 500 \mathrm{Mev}$. The corresponding value of $\mathrm{E}=9.66$ will be used in the calculation in Section IIID.

As a final kinematical consideration let us determine the possible range of $\lambda$ for a given $E$ and $T_{L^{\circ}}$. In order to derive this range, note the relationship of $\lambda, E$, and $\Delta^{2}$ to laboratory quantities, denoted by the subscript $\mathrm{L}$ :

$$
\begin{aligned}
& \lambda=2 T_{L} \epsilon_{2 L}(I-\cos \psi) \\
& E^{2}=M^{2}-\lambda+2 M\left(T_{L}-\epsilon_{2 L}\right) \\
& \Delta^{2}=2 M_{2 L}\left(E_{2 L}-M\right) .
\end{aligned}
$$

Here $\psi$ is the laboratory angle between the initial and final electron directions. In Eq. (2.15) the mass of the electron has been neglected compared to its momentum. From Eqs (2.15) and (2.16) we find

$$
E^{2}=M^{2}+2 M T_{L}-\lambda\left[1+\frac{M}{T_{L}(I-\cos \psi)}\right] .
$$

For a given $\lambda$, the maximum value of $\mathrm{E}^{2}$ is obtained for backward scattering, $\psi=\pi$. Conversely; $\psi=\pi$ also gives the maximum value of $\lambda$ permitted for a given $\mathrm{E}$ and $\mathrm{T}_{\mathrm{L}}$ (see Fig. 7). By increasing $\mathrm{T}_{\mathrm{L}}$ one can obtain higher values of $\lambda$. 


\section{ESTIMATE OF ELECTROPRODUCTION CROSS SECTION}

\section{A. General.}

In order to assess the difficulty of carrylng out the proposed extrapolation, one must estimate the electroproduction cross section as a function of $\cos \theta$, with the psrameters $\lambda, E,{ }_{\mathrm{T}}$, and $\varnothing$ held fixed. A general treatment of the electroproduction problem has been given by Dalitz and Yennie ${ }^{13}$ (hereafter DY). The most recent calculation, bised on the photoproduction theory of Chew, Goldberger, Iow, and Nambu, 9 was made by FNW. In this section a treatment will be given which relies heavily on the aforementioned papers; but which treats somewhet differently the corrections due to the finite mass of the nucleon.

Define the $\mathrm{T}$ matrix

$S_{f i}=\delta_{f i}-i \delta^{4}\left(p^{0}+q+s^{0}-p-s\right)\left(\frac{m^{2} M^{2}}{\omega_{2} E_{1} E_{2} \epsilon_{1} \epsilon_{2}}\right)^{1 / 2} \cdot T_{f i}$

The $T$ matrix element can be expressed in terms of the current $j$ associated with the transition from nucleon to finsl pion-nucleon state:

$$
T=\frac{e g_{r}}{(2 \pi)^{T / 2}} j \circ \varepsilon
$$

This form applies to both electroproduction and photoproduction. In the latter case, $\epsilon$ is proportional to the polarization vector; in the former, $\epsilon$ has to lowest order in e the value

$$
\epsilon_{\mu}=\frac{e \bar{u}\left(s^{0}\right) \gamma_{\mu} u(s)}{\lambda} .
$$

The conservation of charge, expressed as a continuity equation in momentum space, requires that 


$$
\mathrm{k} \cdot \epsilon=\mathrm{k} \cdot \mathrm{j}=0 .
$$

By means of these relations the time components of $j$. and $\epsilon$ can be expressed in terms of their space components. This will prove convenient in adapting photoproduction results to electroproduction. The relation between the two processes. becomes clear if one separates the space part $\vec{j}$ of the current into a longitudinal component

$$
\overrightarrow{\mathrm{j}}_{l} \equiv \overrightarrow{\mathrm{k}}(\overrightarrow{\mathrm{k}} \circ \overrightarrow{\mathrm{j}}) /\left.\mathrm{|} \overrightarrow{\mathrm{k}}\right|^{2}
$$

and a transverse component $\vec{j}_{t}$ such that

$$
\vec{j}=\vec{j}_{l}+\vec{j}_{t}
$$

Then using (3.4) one can write

$$
\mathrm{T} \sim j \circ \epsilon=\lambda \mathrm{k}_{0}{ }^{-2} \vec{j}_{\boldsymbol{l}} \cdot \vec{\epsilon}=\vec{j}_{t} \cdot \vec{\epsilon}
$$

This result exhibits clearly the difference between the electropion and photopion-production matrix elements: Whereas photoproduction depends only on the transverse current, having $\lambda=0$; electroproduction depends also on the longitudinal current.

Following DY, (3.7) can be written in the compact form

$$
j \circ \epsilon=\vec{m} \cdot \vec{\epsilon},
$$

where $\overrightarrow{\mathrm{m}}_{t} \equiv \vec{j}_{t}$, and $\overrightarrow{\mathrm{m}}_{\ell} \equiv \lambda \mathrm{k}_{0}^{-2} \vec{j}_{\ell}$

Remembering the definition of dP given by (2.4), one can write:

$$
\frac{\mathrm{d} \sigma}{\mathrm{dP}}=\frac{2(2 \pi)^{2} \mathrm{M} \mathrm{m}^{2}}{\mathrm{~s}_{\mathrm{L}}} \underset{\mathrm{N}_{\mathrm{g}} \mathrm{e}}{\mathrm{s}}|\mathrm{T}|^{2}
$$


where the sum is to be taken over initial and final spin states of the electron and nucleon. The sum over electron spins can be performed immediately, giving ${ }^{*}$

$m^{2} \sum_{N, e}|T|^{2}=\frac{8 \alpha^{2} g^{2}}{(2 \pi)^{4} \lambda^{2}} \sum_{N} j_{\mu}^{\dagger} j_{\nu} L^{\mu \nu}$,

where the sum $\Sigma_{\mathrm{NN}}$ is to be taken over the initial and final nucleon states, and where

$$
\begin{aligned}
L^{\mu \nu} & \equiv m^{2} \sum_{e} \vec{u}(s) r^{\mu} u\left(s^{\nu}\right) \bar{u}\left(s^{0}\right) r^{\nu} u(s) \\
& =2 \cdot s^{\mu} s^{\nu}-s^{\mu}: k^{\nu}-s^{\nu} k^{\mu}-\frac{1}{2} \lambda g^{\mu \nu} .
\end{aligned}
$$

The cross section can then be written simply

$$
\begin{aligned}
& \frac{d \sigma}{d P}=\frac{\alpha^{2} g^{2}}{\pi^{2} M s_{L} \lambda}\left(\frac{E}{M}\right)^{2} \varnothing^{\gamma} \\
& \text { where } \varnothing^{\prime} \text { is defined by }
\end{aligned}
$$

$\Phi=\lambda^{-1}(2 M)^{2}(M / E)^{2} \sum_{\mathbb{N}} j_{\mu}^{+} j_{\nu} I^{\mu \nu}$.

If $j$ is known, $\varnothing$ can be evaluated by using (3.12). It is sometimes convenient, however; to use the form (3.8), which lesds to the expression

The summation convention is used throughout. Greek indices are summed from 0 to 3; Latin indices, from 1 to 3 . 


$$
\begin{aligned}
& \lambda_{i} \Phi=\left(2 M^{2} / E\right)^{2} \sum_{\mathbb{N}} L_{i, j} m_{i}^{+} m_{j} \\
& =\left(2 M^{2} / E\right)^{2} \sum_{\mathbb{N}}^{\Sigma}\left\{2\left|\vec{s} \cdot \vec{m}_{t}\right|^{2}+\frac{1}{2} \lambda_{b}\left|\vec{m}_{t}\right|^{2}\right. \\
& +2 \operatorname{Re}\left(\vec{s} \circ \vec{m}_{t}+\right)(\vec{k} \cdot \vec{m}) \frac{|\vec{s}|^{2}-\left|\vec{s}^{0}\right|^{2}}{|\vec{k}|^{2}} \\
& \left.+|\overrightarrow{\mathrm{k}} \cdot \overrightarrow{\mathrm{m}}|^{2} \frac{1}{2} \frac{\mathrm{k}_{0}^{2}}{|\overrightarrow{\mathrm{k}}|^{2}}\left[-1+\frac{\left(|\overrightarrow{\mathrm{s}}|+\left|\overrightarrow{\mathrm{s}^{\prime}}\right|\right)^{2}}{|\overrightarrow{\mathrm{k}}|^{2}}\right]\right\} .
\end{aligned}
$$

The foregoing formulation of electroproduction is completely general, except for the excellent approximation of neglecting contributions of higher order in $\alpha$. In order to handle the remaining problem of obtaining an expression for the nucleon-meson current matrix element $j$, it will be necessary to invoke much poorer approximations.

\section{B. Dispersion Theory of Photoproduction and Electroproduction.}

The most successful calculation of photoproduction, given in CGIN, is based on the method of spectral representations. This calculation was extended to electroproduction by FIN. At the values of the experimental parameters suggested in Section II $C$, namely $\mathrm{T}_{\mathrm{L}}=700 \mathrm{Mev}$ and $\mathrm{k}_{\mathrm{ph}}=500$ Mev, the approximate solutions of the dispersion equations given in CGLN cannot be expected to be very accurate. The solutions are based on neglecting terms of higher than first order in $\mu / M, \omega / M$, and $|\vec{k} \cdot| / M$. When $\mathrm{k}_{\mathrm{ph}}=500$ Mev and $\lambda=10,|\overrightarrow{\mathrm{k}}| / \mathrm{M}=0.56$. An indication of the accuracy of the CGLN formulas can be obtained by comparison with photoproduction data. Although photoproduction of neutral pions from protons 
is in excellent agreement with the CGLN theory up to $450 \mathrm{Mev}$ lab energy, 14 Uretsky et al find a definite disagreement in positive pion production at $290 \mathrm{Mev}^{15}$ The disagreement becomes worse as the energy increases; at $400 \mathrm{Mev}$ the measured value in the backward direction is only about half as large as the theoretical value. A sumary of the comparison of theory and experiment in positive pion production is given by FIg. 8 of the paper by Lazarus, Panofsky, and Tangherlini。 16 The lack of quantitative success of the photoproduction theory at high energies need not discourage us much here. The theory gives a semi-quantitative fit, which should be quite adequate for the purpose of estimating the difficulty of the proposed extrapolation.

Let us now consider those aspects of the dispersion theory of electroproduction which are most important for our calculation, referring. the reader to FNW for a more detailed account of the mathematical procedure. The existence of a dispersion relation for the electroproduction scattering amplitude has been proved by Oehme and Taylor. ${ }^{17}$ The dispersion relation expresses the scattering amplitude as the sum of an integral over a spectrum of masses of possible intermediate states plus a term that is just the renormalized Born approximation with appropriate form factors. The Born term contribution to $j$ is: *

$$
\begin{aligned}
& \text { The notation is such that }(r \circ p-m) u(p)=0 \text { and } \\
& r^{5}=i r_{1} r_{2} r_{3} r_{4} \text {. }
\end{aligned}
$$




$$
\begin{aligned}
& -25 \\
& \sqrt{2} e j_{B}^{\nu}=\bar{u}\left(p^{0}\right)\left\{i r_{5} \frac{e F_{\pi}(2 q-k)^{\nu}}{\Delta^{2}+\mu^{2}} \frac{\left[\tau_{\alpha}, \tau_{3}\right]}{2}\right. \\
& -i \gamma_{5} \frac{\gamma \cdot(p+k)+M}{(p+k)^{2}-M^{2}} \tau_{\alpha}\left[\frac{e^{s}+\tau_{3} e^{v}}{2} \gamma^{\nu}+\frac{\mu^{s}+\tau_{3} \mu^{v}}{2} \sigma^{\nu \rho} k_{\rho}\right] \\
& \left.=\left[\frac{e^{s}+\tau_{3} e^{v}}{2} \gamma^{\nu}+\frac{\mu^{8 s}+\tau_{3} \mu^{0 v}}{2} \sigma^{\nu \rho} k_{\rho}\right] \tau_{\alpha} \frac{r \cdot(p-q)+M}{(p-q)^{2}-M^{2}} i \gamma_{5}\right\} u(p),
\end{aligned}
$$

where

$$
\left.\begin{array}{l}
e^{v, s}(\lambda)=e\left[F_{1}^{p}(\lambda) \pm F_{1}^{n}(\lambda)\right] \\
\mu^{\prime v, s}(\lambda)=\mu_{p}{ }^{p} F_{2}^{p}(\lambda) \pm \mu_{n} F_{2}^{n}(\lambda)
\end{array}\right\} \quad \begin{aligned}
& \text { - for } v \\
& \text { + for } s .
\end{aligned}
$$

The form factors $F_{1,2}$ are the customary nucleon form factors introduced in connection with electron-nucleon scattering, ${ }^{18}$ and. $\mu_{\mathrm{p}}{ }^{\prime} \approx 1.78 \mathrm{e} / 2 \mathrm{M}$ is the anomalous part of the proton magnetic moment. The first term in Eq. (3.16), arising from the diagram of Fig. 3, contains the pole on which the extrapolation is based. Note the presence of the pion form factor, which was set equal to unity in FNW. This first term is the meson current contribution, wheress the second and third terms, arising from the diagrams of Fig. 8, are the nucleon current contribution.

The Born terms $j_{B}$ do not satisfy the continuity equation (3.4). One finds

$$
k \cdot j_{B}=(e-\sqrt{2})^{-1} \bar{u}\left(p^{0}\right) i: \gamma_{5} \frac{1}{2}\left[\tau_{\alpha}, \tau_{3}\right]\left(e F_{\pi}-e^{v}\right) u(p) .
$$


As pointed out in FNW, this situation can be corrected by the formal addition to $\left.j_{B}\right)$ of the term

$$
\frac{k^{\nu}}{\lambda} \frac{1}{e \sqrt{2}} \bar{u}\left(p^{p}\right) \text { i } r_{5} \frac{1}{2}\left[\tau_{\alpha}, \tau_{3}\right]\left(e F_{\pi}-e^{v}\right) u(p) \text {. }
$$

Since $\lambda \equiv-k^{2}$, this term will cancel the nonzero contribution of $\mathrm{Eq} \cdot(3.17)$ : Moreover, because of $\mathrm{Eq} \cdot(3.4)$, the contribution of $\mathrm{Eq} \cdot(3.18)$ to electroproduction; $1_{\circ} e_{\circ}$, to $j \cdot \epsilon$, is zero. This formal device, or an equivalent one, is nevertheless necessary if one wishes to make use of formulae such as Eqs. (3.7) and (3.15) which simplify the computation. Specializing now to the production of positive pions from protons, we consider first the contribution to $j_{B}$ of the terms proportional to e (rather than to $\mu$ ). These are:

$$
\begin{aligned}
j_{B, e} \nu=\bar{u}\left(p^{0}\right)\left\{1 \gamma_{5} \frac{F^{\prime}(2 q-k)^{\nu}}{\Delta^{2}+\mu^{2}}-1 r_{5} \frac{r \cdot(p+k)+M}{E^{2}-M^{2}} r^{\nu} F_{I}^{p}\right. \\
\\
\left.\quad-r^{\nu} F_{I} n \frac{r \cdot(p-q)+M}{(p-q)^{2}-M^{2}} i r_{5}\right\} \therefore
\end{aligned}
$$

The form factor $F_{I}{ }^{p}$. Is quite well known from the electron-proton scattering experiments by Hofstadter and collaborators. For $\mathrm{F}_{1}{ }^{\mathrm{n}}$, the assumption $F_{1}^{n}=0$ will be made. Present experimental results indicate that no large error is made by: this assumption at the vilue $\lambda=10 \mu^{2}:\left(=5 \times 10^{26} \mathrm{~cm}^{-2}\right){ }^{19}$ 
Having set $F_{I}{ }^{n}=0$, let us express the remsining terms in Eq. (3.19) by means of Pauli spinors, using the representation.

$$
\left.u^{r}(p)=v_{p}\left(\cdot 1+r_{5} \vec{\sigma}_{\cdot} \vec{R}_{p}\right) \mid r\right) \text {, }
$$

where $|r\rangle$ is now a two-component Pauli spinor characteristic of spin state $r$, where $v_{p} \equiv\left[\left(M+E_{p}\right) / 2 M\right]^{I / 2}$ and $\vec{R}_{p} \equiv \vec{p} /\left(M+E_{p}\right)$. With this substitution, the Born terms associated with $e$ become, in the $\vec{p}^{0}+\vec{q}=0$ system,

$$
\begin{aligned}
& \vec{j}_{B, e}=i v_{1} v_{2}\langle f|\left\{\frac{F_{1}^{p} \vec{\sigma}}{E+M} \cdot \frac{F_{\pi}(2 \vec{q}-\vec{k}) \cdot \vec{\sigma} \cdot\left(\vec{R}_{q}-\vec{R}_{k}\right)}{\Delta^{2}+\mu^{2}}\right. \\
& \left.-\frac{F_{1}^{p}\left(\vec{\sigma} \cdot \vec{R}_{q}\right) \cdot \vec{\sigma}\left(\vec{\sigma}_{0} \vec{R}_{k}\right)}{E-M}\right\}|i\rangle
\end{aligned}
$$

In the dispersion-theory analysis of photoproduction in CGIN, an expansion $\cdots$ in powers of $\mathrm{m} / \mathrm{M}$ is made, and only the zeroth and first-order terms are kept: The zeroth-order term in Eq. (3.21) is

$\vec{j}_{B, e} 0=\frac{i}{2 M}\left\langle f\left|\left\{F_{1}^{p} \vec{\sigma}-F_{\pi} \frac{(2 \vec{q}-\vec{k}) \vec{\sigma} \cdot(\vec{q}-\vec{k})}{(\vec{q}-\vec{k})^{2}+\mu^{2}}\right\}\right| i\right\rangle$.

This term is an important contribution to the expression (14) of FNW for electroproduction. Additional contributions come from the first-order terms in $1 / \mathrm{M}$ of Eq. (3.21), as well as terms arising from the nucleon magnetic moment part of $j_{B}$. The most important additional term is the magnetic-dipole amplitude leading to a resonant finsl $P$ state with 
total angular momentum $3 / 2$ and isotopic spin $3 / 2$ 。 The resonant amplitude arises primarily from the nucleon moments. The meson current also produces resonant terms, proportional to the factors $F_{M^{\prime}}, F_{Q^{\prime}}$ and $F_{L}$ of $E q_{0}$ (13) of FNW but these are much smaller for the case to be calculated here and will be neglected. The resonant magnetic dipole part of $\vec{j}$ for positive pion production is then

$\vec{j}_{M}=-\frac{E}{M} \frac{D}{2 M}\langle f|[2 \vec{q} \times \vec{k}-i(\vec{\sigma} \cdot \vec{k}) \vec{q}+i \vec{\sigma}(\vec{q} \cdot \vec{k})]| i\rangle$,

where.

$$
D \equiv \frac{\mu^{v}(\lambda)}{6 f^{2}|\vec{q}|^{3}} e^{i \delta_{33}} \sin \delta_{33} .
$$

The phase $\delta_{33}$ is the pion-nucleon scattering phase shift at the corresponding energy.

Several features of $\vec{j}_{M}$ deserve some comment. First, all momenta must be evaluated in the $\vec{p}^{\prime}+\vec{q}^{0}=0$ system, which corresponds to the barycentric system in photoproduction. In a more general Lorentz frame, the multipole expansion ${ }^{20}$ on which the relations in CGLN depend is not valid, because another vector in addition to $\vec{q}, \vec{k}, \vec{\sigma}$, and $\vec{\epsilon}$ (for) instance, the total momentum of the system) would appear in the expansion. Second, the factor $\mathrm{E} / \mathrm{M}$ in Eq. (3.23) is necessary. because the CGIN formulae include a factor $\mathbb{M} / E$ (see CGLN, Formula 7.1 ) from the photoproduction phase space. Since the current $j$, as defined by

Corrections to the Born approximation values of the S-wave and small Powave amplitudes will also be neglected. 
Eqs. (3.1) and (3.2), does not contain phase-space factors, the factor $\mathrm{M} / \mathrm{E}$ is necessary. Third, the quantity $\mu^{\mathrm{v}}$ in Eq. (3.23) is defined as

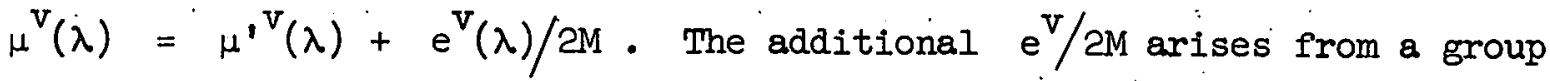
of the $I / M$ corrections to the zeroth approximation to the e Born terms, Eq. $(3.22)$.

The two terms $\vec{j}_{\mathrm{Be}} 0$ and $\vec{j}_{\mathrm{M}}$ are the most important contributions to the electroproduction amplitude $j$. To estimate the cross section on the basis of these two terms alone would, however, involve the neglect of terms such as $\omega / M$ and $|\vec{k}| / M$ which are not negligible. The expressions of CGLN and FNW include correction terms which arise from evaluating Eq. (3.16) in first order in $1 / \mathrm{M}$. The result for positive-pion production (FNW Eq. (14)) is, after making the approximation discussed above of setting $F_{Q}=F_{L}=F_{M}=F_{I}^{n}=0$ :

$2 M \vec{j} \cdot \vec{\epsilon}=2 M\left(\vec{j}_{B e} 0+\vec{j}_{M}\right) \cdot \vec{\epsilon}-i \vec{\sigma} \cdot \vec{\epsilon} \mu \frac{\vec{k}^{2}}{\omega^{*}}$

$$
-\frac{i \vec{\sigma} \cdot \vec{q} \times(\vec{k} \times \vec{\epsilon})}{\omega^{*}} \mu^{s}-\frac{i \text { e } F_{1}^{p}(\vec{\sigma} \cdot \vec{q})(\vec{k} \cdot \vec{\epsilon})}{2 M \omega^{*}},
$$

plus a term coming from Eq. (3.18), which was inserted to satisfy the continuity equation. Here $\omega^{*} \equiv \mathrm{E}-\mathrm{M}$.

An alternative procedure, which will be employed here for including recoil corrections, is to replace the zeroth approximation $\vec{j}_{\mathrm{Be}} 0$ by the complete covariant expression $\vec{j}_{\mathrm{Be}}$ given by Eq. (3.19) or (3.21); i.e., set

$$
\vec{j}=\vec{j}_{\mathrm{Be}}+\vec{j}_{\mathrm{M}} \text {. }
$$


The first-order term in $I / M$ of $\vec{j}_{\mathrm{Be}}$ includes the last term of Eq. (3.24), and includes that portion of the second and third terms which arises from the Dirac moment of the nucleon. If we assume, consistent with electronucleon scattering experiments, ${ }^{19}$ that $F_{1}^{P}=F_{2}^{P}=F_{2}{ }^{n} \equiv F_{N}$, we find that

$$
\mu^{s}=\left(I+g_{p}^{0}+g_{n}\right) F_{N} e / 2 M
$$

Since $g_{p}{ }^{0}+g_{n}=-0.13$. we see that a relatively small error will be made by neglecting the anomalous moment part.

Thus expression (3.25) includes, to a good approximation, the $1 / \mathrm{M}$ terms of $(3.24)$. In addition, by avoiding the expansion of $\vec{j}_{\mathrm{Be}}$ in powers of $1 / M$, it includes some of the higher order corrections which have been dropped in $(3.24)$. One might hope that these corrections which have been included are the more important ones. The magnetic dipole amplitude $\vec{j}_{M}$ already includes recoil corrections to some extent when one uses experimental values from pion-nucleon scattering for the phase shift. In fact, the CGLN formula for photoproduction of neutral pions, which is dominated by $\vec{j}_{\mathbb{M}}$, has been shown to agree well with the experimental data ${ }^{15}$ up to 450 Mev photon laboratory energy.

In order to gain further confidence in the use of the amplitude $\vec{j}_{M}$ at energies well above resonance and at values of $\lambda$ up to the value $\lambda=10$ to be used here, let us examine the portion of the CGIN theory that gives rise to the specific form of $\vec{j}_{M^{*}}$ First we must define the notation used for photoproduction by CGIN. Denoting the complete photoproduction amplitude by $7_{\gamma},:$ i.e., 


$$
\frac{d \sigma}{d \Omega}=\Sigma \frac{|\vec{q}|}{|\vec{k}|}\left|\left\langle f\left|\exists_{\gamma}\right| i\right\rangle\right|^{2},
$$

one can write, neglecting $D$ waves and higher, 20

$$
\begin{aligned}
\vec{f}_{\gamma}= & i \vec{\sigma} \cdot \vec{\epsilon} E_{S} \frac{1}{2}+\frac{1}{|\vec{q}||\vec{k}|}[\vec{q} \cdot \vec{k} \times \vec{\epsilon}+i \vec{\sigma} \cdot \vec{q} \times(\vec{k} \times \vec{\epsilon})] M_{P} \frac{1}{2} \\
& +\frac{1}{|\vec{q}||\vec{k}|}[2 \vec{q} \cdot \vec{k} \times \vec{\epsilon}-i \vec{\sigma} \cdot \vec{q} \times(\vec{k} \times \vec{\epsilon})] M_{P} \frac{3}{2} \\
& +\frac{3 i}{|\vec{q}||\vec{k}|}[(\vec{\sigma} \cdot \vec{k})(\vec{q} \cdot \vec{\epsilon})+(\vec{\sigma} \cdot \vec{\epsilon})(\vec{q} \cdot \vec{k})] E_{P} \frac{3}{2}
\end{aligned}
$$

The energy-dependent amplitudes $M_{1 j}$ and $E_{1 j}$ refer to production by magnetic and electric radiation, respectively, leading to final states of orbital angular momentum 1 and total angular momentum $j$. Each amplitude can be further decomposed into a sum of multiples of the three lndependent isotopic spin amplitudes, defined as

$$
\begin{aligned}
& t_{\beta}^{(+)} \equiv \frac{1}{2}\left[\begin{array}{ll}
T_{\beta}, & \left.T_{3}\right]_{+}
\end{array}\right. \\
& \ell_{\beta}^{(-)} \equiv \frac{1}{2}\left[\tau_{\beta}, \tau_{3}\right] \\
& t_{\beta}^{(0)} \equiv \tau_{\beta} \text {, }
\end{aligned}
$$

where $\beta$ is the isotopic spin index of the pion. For positive pion production, the matrix elements of $f^{( \pm, 0)}$ are $f^{(+)}=0$, $f^{(-)}=f^{(0)}=\sqrt{2}$. Now each amplitude $M_{1 j}, E_{1 j}$, can be decomposed 
into a sum of multiples of $\ell_{\beta}( \pm, 0)$; for example,

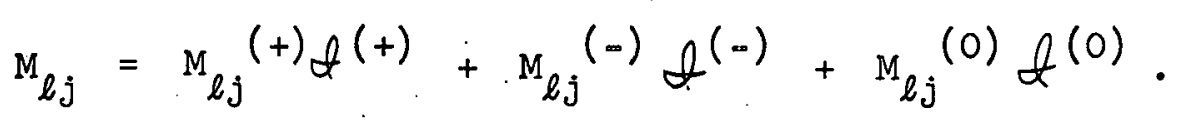

Comparison of Eqs. (3.23) and (3.27) shows that the photoproduction amplitude corresponding to $\vec{j}_{\mathrm{M}}$ is $\mathrm{M}_{\mathrm{P}}{ }^{( \pm)}$. The important conclusion in CGLN concerning this amplitude is that the part of $\mathrm{M}_{\mathrm{P}} 3^{( \pm)}$. which is induced by the nucleon magnetic moment (this is the dominant part) is proportional to the corresponding. Pawave pion-nucleon scattering amplitude. To reproduce the precise statement of proportionality, let us note the definition of amplitudes for pion-nucleon scattering in the barycentric system:

$$
\frac{\mathrm{d} \sigma}{\mathrm{d} \Omega}=\Sigma\left|\left\langle\mathrm{f}\left|\mathrm{J}_{\pi}\right| i\right\rangle\right|^{2},
$$

where, neglecting $\mathrm{D}$ waves and higher,

$$
\begin{aligned}
\exists_{\pi}=f_{S \frac{1}{2}}+\frac{f_{P} \frac{1}{2}}{|\vec{q}|^{2}}\left(\vec{q}_{2} \cdot \vec{q}_{1}+i \cdot \vec{\sigma}_{q_{2}} \times \vec{q}_{1}\right) \\
+\frac{f_{P \frac{3}{2}}}{|\vec{q}|^{2}}\left(2 \vec{q}_{2} \cdot \vec{q}_{1}-i \vec{\sigma}_{q_{2}} \times \vec{q}_{1}\right)
\end{aligned}
$$

The isotopic spin decomposition has the form

$$
\dot{f}_{\ell j}=\delta_{\alpha \beta} f_{\ell j}^{(+)}+\frac{1}{2}\left[\tau_{\beta}, \tau_{\alpha}\right] f_{\ell j}^{(-)}
$$

where $\alpha$ and $\beta$ are the isotopic spin indices of the initial and final mesons. Then in terms of these amplitudes the solution found to lowest 
order in $1 / M$ in CGLN (Equation 13.1) for the nucleon moment part of $\mathrm{M}_{\mathrm{P}} \frac{3}{2}^{( \pm)}$ is

$$
\frac{1}{q \mathrm{k}} \mathrm{M}_{\mathrm{p}} \frac{3}{2}^{( \pm)}=\frac{\mu_{\mathrm{p}}-\mu_{\mathrm{n}}}{2 \mathrm{f}|\overrightarrow{\mathrm{q}}|^{2}} \mathrm{f}_{\mathrm{p}}{ }^{( \pm)} \text {. }
$$

The definition (3.30) of $\mathrm{f}_{\ell j}$, together with the unitarity of the S matrix, implies that $f_{\mathrm{P}} \frac{3}{2}^{(-)}$can be written (keeping only the $\mathrm{T}=\frac{3}{2}$ state)

$$
f_{P} \frac{3}{2}^{(-)}=-\frac{1}{3} e^{i \delta_{33}} \sin \delta_{33} /|\vec{q}| .
$$

The relations (3.31) and (3.32) show the origin of the expression (3.23) for $\vec{j}_{M}$, except for the form factors and normalization peculiar to the electroproduction case.

If we wish to use the form of $\vec{j}_{M}$ given in (3.23) at energies well above the (3.3) resonance energy, the important question is: How large a deviation can we expect from the simple proportionality (3.31) when higherorder terms in $1 / \mathrm{M}$ become appreciable? A partial answer to this question can be obtained by considering the deviation from Eq. (3.31) of the Bornterm contributions to $\mathrm{M}_{\mathrm{P}} \frac{3}{2}$, and $\mathrm{f}_{\mathrm{P}} \frac{3}{2}$. By the straightforward but lengthy process of projecting out the $\mathrm{P} \frac{3}{2}$. part of the pion-nucleon-scattering Born approximation, one obtains, neglecting $D$ waves and higher,

$$
f_{P} \frac{3}{2}^{( \pm), B}= \pm \frac{2 M g^{2} v_{2}^{2} \omega^{*}|\vec{q}|^{2}}{3 E\left(2 E_{2} \omega_{2}-1\right)^{2}},
$$

where $E_{2} \equiv\left(|\vec{q}|^{2}+M^{2}\right)^{1 / 2}, \omega_{2} \equiv\left(|\vec{q}|^{2}+1\right)^{1 / 2}, \quad E=\omega_{2}+E_{2}$, $\omega^{*}=E-M$, and $V_{1,2}=\left[\left(M+E_{1,2}\right) / 2 M\right]^{I / 2}$. Expanding in powers 
of $1 / M$, one finds

$$
f_{P} \frac{3}{2}^{( \pm), B}= \pm \frac{2}{3} \frac{f^{2}|\vec{q}|^{2}}{\omega^{*}}+o\left(\frac{1}{M^{2}}\right) .
$$

The electroproduction and photoproduction Born terms are of two kinds: those proportional to the electric charge and those proportional to the anomalous magnetic moment. For electroproduction, which reduces e essily to photoproduction by setting $\lambda=0$, the contribution to $\mathrm{M}_{\mathrm{P}} \frac{3}{2}$ of the nucleon current part of the electric Born terms is

$$
\frac{M_{p} 3^{( \pm), B, e}}{q k}= \pm \frac{e^{v} f}{3} F_{p} \frac{3}{2}
$$

where

$$
F_{P} \frac{3}{2} \equiv \frac{M^{2} V_{2} V_{1}}{E}\left[\frac{\omega^{*}}{\left(2 E_{2} \omega_{2}-1\right)^{2}}+\frac{1}{\left(E_{1}+M\right)\left(2 E_{1} \cdot \omega_{2}-1\right)}\right] \text {. }
$$

An expension in powers of $1 / M$ gives

$$
\frac{\mathrm{M}_{\mathrm{P} \frac{3}{2}}( \pm), B, e}{|\overrightarrow{\mathrm{q}}||\overrightarrow{\mathrm{k}}|}= \pm \frac{\mathrm{e}^{\mathrm{V}}}{2 \mathrm{M}} \frac{\mathrm{f}}{3 \omega^{*}}\left(1-\frac{\omega^{*}}{4 \mathrm{M}}\right)+O\left(\frac{1}{\mathrm{M}^{3}}\right)
$$

The anomalous-moment Born-term contribution is, for electroproduction,

$$
\frac{M_{P} \frac{3}{2}^{( \pm), B, \mu}}{|\vec{q}||\vec{k}|}= \pm \frac{\mu^{\prime v} g v_{1} v_{2}}{3 E\left(2 E_{1} \omega_{2}-I\right)}\left[1+\frac{k_{0}}{2\left(E_{1}+M\right)}+\frac{k_{0} \omega_{2}}{2\left(2 E_{1} \cdot \omega_{2}-I\right)}\right],
$$


which becomes, in a $1 / M$ expansion,

$$
\frac{M_{P} \frac{3^{2}}{( \pm), B, \mu}}{|\vec{q}||\vec{k}|}= \pm \frac{\mu^{, v} f}{3 \omega^{*}}+o\left(\frac{I}{M^{2}}\right)
$$

Recalling that $\mu^{\mathrm{v}}(\lambda) \equiv \mu^{\prime} \mathrm{v}(\lambda)+e^{\mathrm{V}}(\lambda) / 2 \mathrm{M}$, we see that the simple proportionality,

$$
\begin{aligned}
\frac{1}{|\vec{q}||\vec{k}|}\left(M_{P} \frac{3}{2}^{( \pm), B, e}+M_{P} \frac{3}{2}^{( \pm), B, \mu}\right) & \equiv \frac{1}{|\vec{q}||\vec{k}|} M_{P} \frac{3}{2}^{( \pm), B} \\
& =r \frac{\mu(\lambda)}{2 f|\vec{q}|^{2}} f_{P} \frac{3}{2}
\end{aligned}
$$

is exact to order $I / M$ with $r=1$. Calculating the exact $r$ from Eqs. $(3.33),(3.35),(3.37)$, and $(3.39)$, one finds for photoproduction at 260and 400-Mev laboratory energies that $r=0.990$ and 1.007 , respectively, whereas for electroproduction at $\mathrm{k}_{\mathrm{ph}}=500 \mathrm{Mev}$ and $\lambda=10, \quad r=0.932$. Thus in photoproduction the simple proportionality of the Born terms is retained well above the (3.3) resonance energy; even for electroproduction, up to at least the stated values of $k_{p h}$ and $\lambda$, the value of $r$ can be set equal to unity with sufficient accuracy for our purposes. The expression (3.23) for $\vec{j}_{M}$ will therefore be used as it stands. One further comment should be made about the expression (3.25) which will be used for $\vec{j}$, namely, that the inclusion of both $\vec{j}_{B e}$ and $\vec{j}_{M}$ results in counting one term twice. We have seen above in Eq. (3.35) 
that $\vec{j}_{\mathrm{Be}}$ contributes to the amplitude $\mathrm{M}_{\mathrm{P}} \frac{3}{2}( \pm), \mathrm{B}, \mathrm{e}$. This contribution is included in $\vec{j}_{M}$ and should therefore be subtracted cut of $\vec{j}_{B e}$. Equation (3.35) shows that the quantity that should be subtracted from $\vec{j}$ is

$-\frac{e^{v} E}{6 M^{2}} F_{P} \frac{3}{2}\langle f|[2 \vec{q} \times \vec{k}-i(\vec{\sigma} \cdot \vec{k}) \vec{q}+i(\vec{q} \cdot \vec{k}) \vec{\sigma}]| i\rangle$.

Numerically this term proves to be 20 more important in the case to be considered than many terms already neglected and will therefore be neglected also.

\section{Calculation of the Photoproduction Cross Section.}

The formula (3.25) for $\vec{j}$ developed in the previous section avoids. expanding the electric Born terms in powers of $1 / M$ and may therefore include nucleon-recoil effects more accurately than the CGLN formula. Since ciefinite disagreement has been observed in positive-pion production between the CGLN formula and experinent, ${ }^{15,16}$ a calculation has been made at a laboratory energy of $400 \mathrm{Mev}$ to see if Formuia (3.25) leads to better agreement. Unfortunately this does not seem to be the case. The calculation, which will be described in this section, led to an angular distribution almost identical to that predicted by the CGLN formula. Retaining essentially the CGLil notation, one finds from Eq. (3.25) that the amplitude for positive-pion production is: 
$\frac{f_{r}^{(-)}+f_{r}^{(0)}}{e f}=\frac{v_{1} v_{2}}{1+\frac{\omega^{*}}{M}}\left[\frac{i \vec{\sigma} \cdot \vec{\epsilon}}{1+\frac{\omega^{*}}{2 M}}+\frac{i 2(\vec{q} \cdot \vec{\epsilon}) \vec{\sigma} \cdot\left(\frac{\vec{k}}{v_{1}^{2}}-\frac{\vec{q}}{v_{2}^{2}}\right)}{(\vec{q}-\vec{k})^{2}+1-\left(E_{2}-E_{1}\right)^{2}}\right.$

$$
\begin{aligned}
& \left.-\frac{i(\vec{\sigma} \cdot \vec{q})(\vec{\sigma} \cdot \vec{\epsilon})(\vec{\sigma} \cdot \vec{k})}{2 M \omega^{*} v_{2}^{2} v_{1}^{2}}\right] \\
& +i \vec{\sigma} \cdot \vec{\theta}\left[i\left(\frac{2}{3} \delta_{1}+\frac{1}{3} \delta_{3}\right) \mathrm{F}_{S}+\omega *^{2} N{ }^{(-)}\right] \\
& +i(\vec{\sigma} \cdot \vec{\epsilon})(\vec{q} \cdot \vec{k})\left[-\lambda h h^{(-)}+\frac{l}{3}\left(F_{P} \frac{3}{2}-F_{P} \frac{i}{2}(-)\right)\right. \\
& \left.+\frac{1}{3} i e^{i \delta_{33}} \cdot \sin \delta_{33}\left(F_{Q}-\frac{1}{3} F_{M}\right)\right] \\
& +i(\vec{\sigma} \cdot \vec{k})(\vec{q} \cdot \vec{\epsilon})\left[\lambda h^{(-\infty)}-\frac{1}{3}\left(F_{P} \frac{3}{2}-F_{P} \frac{1}{2}(-)\right)\right. \\
& \left.+\frac{1}{3} i e^{i \delta_{33}} \sin \delta_{33}\left(F_{Q}+\frac{1}{3} F_{M}\right)\right] \\
& +\vec{q} \cdot \vec{k} \times \vec{\epsilon}\left[\lambda h^{(-+)}+\frac{1}{3}\left(2 F_{P} \frac{3}{2}+F_{P} \frac{1}{2}(-)\right)\right. \\
& -\frac{2}{9} i e^{i \delta_{33}} \sin \delta_{33} F_{M} j
\end{aligned}
$$


The only quantity in this equation which has not been defined above or in CGLN is $\mathrm{F}_{\mathrm{P}}{ }_{\frac{1}{2}}{ }^{(-)}$, which is proportional to the contribution of the nucleon current electric Born term to the $\mathrm{M}_{\mathrm{P}} \frac{1}{2}(-)$ amplitude. The value of $\mathrm{F}_{\mathrm{P}} \frac{1}{2}(-)$ can be found straightforwardly but turns out to be quite a long expression. Since $F_{P} \frac{1}{2}$ is only a small correction, it will be sufficient to set ${ }_{\mathrm{P}} \frac{1}{2}{ }^{(-)}=F_{\mathrm{P}} \frac{3}{2}$, an equality which holds only to lowest order in $\mathrm{l} / \mathrm{M}$. The differential cross section can be obtained from Eq. (3.40) via the relation

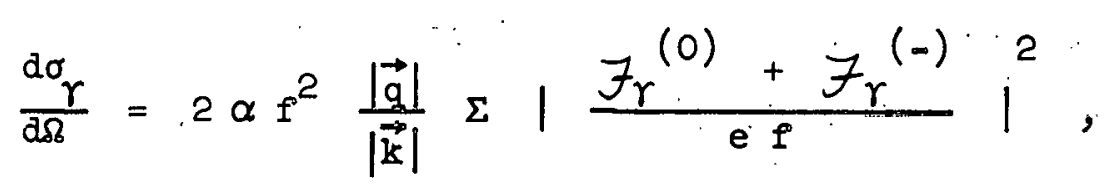

where $\Sigma$ refers to a sum over final nucleon spin states and an average over inftial spin and polarization states.: Comparison calculations of do $/ \mathrm{d} \Omega$ have been carried out at a photon lab energy of $400 \mathrm{Mev}$ by. the use of both Eq. (3.40) and the CGLN Formulae (22.6-7) with the value for $\delta_{33}$ given by the Chew-Low effective-range formula, 9

$$
\cot \delta_{33}=\frac{3 \omega^{*}}{4 f^{2}|\vec{q}|^{3}}\left(I-\frac{\omega^{*}}{\omega_{r}}\right) \text {. }
$$

The phase-shift analysis by Chiu and Lomon ${ }^{21}$ finds the Chew-Low formula accurate up to a scattering energy of $220 \mathrm{Mev}$ ( $370 \mathrm{Mev}$ in photoproduction) if $\omega_{r} \approx 2.1$ and $f^{2}=0.08$. The small Pawave phase shifts, which are not so well known, were set equal to zero in the calculation. The S-wave phase shifts ${ }^{22} \quad \delta_{1}=0.173 q$ and $\delta_{3}=-0.110 q$ were used; and the unknown function $\mathrm{N}^{(-)}$, was set equal to zero. The results and the experimental points of Walker et al. 23 are shown in Fig.9. The use of Formula (3.40) results in no improvement over the CGLN formula for this case. 
Nevertheless the formula developed in this paper may be significantly more accurate in the electroproduction case, where recoil corrections can be more important. The theoretical curves in Fig. 9 agree well with the corresponding curve of Lazarus et al. ${ }^{16}$ The slight difference which exists is probably due to the fact that Lazarus et al. made a different assumption about the small P-wave phase shifts.

D. Calculation of the Electroproduction Cross Section.

Having developed and investigated a formula for $\vec{j}$, we are ready to proceed to a calculation of the electroproduction cross section. This calculation is necessary in order to be able to estimate the experimental accuracy necessary to perform the proposed extrapolation.

Since the machinery for the calculation has been set up in Section III A, we need only insert Formula (3.25) for $\vec{j}$ into Eqs. (3.12) to (3.15). The results will be expressed in terms of the dimensionless quantity $\Phi$, which is related to the cross section by Eq. (3.13). Separating $\varnothing$ into the part coming from $j_{B e}$, from $\vec{j}_{M}$, and from the cross term, we write

$$
\Phi=\Phi_{\mathrm{B}}+\Phi_{\mathrm{M}}+\check{\Phi}_{\mathrm{C}}
$$

The magnetic dipole term $\emptyset_{\mathrm{M}}$ can be evaluated easily by substituting Eq. (3.23) into Eq. (3.15), which gives

$$
\begin{aligned}
\Phi_{M}=|D|^{2}\left\{5|\overrightarrow{\mathrm{q}}|^{2}|\overrightarrow{\mathrm{k}}|^{2}-3(\overrightarrow{\mathrm{q}} \cdot \overrightarrow{\mathrm{k}})^{2}\right. & \\
& \left.+\frac{4}{\lambda}\left[3(\overrightarrow{\mathrm{q}} \cdot \overrightarrow{\mathrm{k}} \times \overrightarrow{\mathrm{s}})^{2}+|\overrightarrow{\mathrm{q}}|^{2}(\overrightarrow{\mathrm{s}} \times \overrightarrow{\mathrm{k}})^{2}\right]\right\},
\end{aligned}
$$

where $D$ is defined by Eq. (3.23'). 
The Born term part of $\varnothing$ can be evaluated covariantly by substituting Eq. (3.19) into Eq. (3.14), by the use of Eq. (3.12)。.The result is:

$$
\begin{aligned}
& \frac{1}{4}\left(\frac{E}{M}\right)^{2} \varnothing_{B}=\frac{\Delta^{2}}{\lambda} \frac{F_{\pi}^{2}}{\left(\Delta^{2}+1\right)^{2}}\left[4(q \cdot s)\left(q \cdot s^{0}\right)-\lambda\right] \\
& +\frac{F_{N}^{2}}{\left(E^{2}: \cdot M^{2}\right)^{2}}\left\{-M^{2}\left(\lambda+\Delta^{2}\right)+(p \cdot k)\left(\Delta^{2}+2 M^{2}\right)-2 M^{2}\left(p^{1} \cdot k\right)\right. \\
& +4 \frac{\Delta^{2}}{\lambda}(p \cdot s)\left(p \cdot s^{0}\right)+2(p \cdot s)\left(p^{0} \cdot s\right)+2\left(p \cdot s^{0}\right)\left(p^{0} \cdot s^{0}\right) \\
& +\frac{4}{\lambda} \cdot\left[2\left(p^{0} \cdot k\right)(p \cdot s)\left(p \circ s^{0}\right)-(p \cdot k)(p \circ s)\left(p^{0} \cdot s^{0}\right)\right. \\
& \left.\left.-(p \cdot k)\left(p \cdot s^{0}\right)\left(p^{\prime} \cdot s\right)\right]\right\} \\
& -\frac{2 F_{N N} F_{\pi}}{\lambda\left(E^{2}-M^{2}\right)\left(\Delta^{2}+1\right)}\left[\left(\Delta^{2}+p^{0} \cdot k\right) h(p)-(p \cdot k) h\left(p^{0}\right)\right] .
\end{aligned}
$$

In this formula the assumption has been made that $F_{I}{ }^{n}(\lambda)=0$, and $F_{1}^{p}(\lambda)=F_{2}^{p}(\lambda)=F_{2}^{n}(\lambda) \equiv F_{N}(\lambda)$, and the abbreviation

$$
h(p) \equiv(2 q-k)_{\mu} p_{\nu} L^{\mu \nu}
$$

has been introduced.

Finally, the cross term is 


$$
\begin{aligned}
& \frac{\mathrm{E} \Phi_{\mathrm{C}}}{2 \mathrm{M} \mathrm{V}_{1} \mathrm{~V}_{2} \operatorname{Re} \mathrm{D}}=-\frac{2 \mathrm{~F}_{\mathrm{N}}}{1+\frac{\omega^{*}}{2 \mathrm{M}}}\left(\overrightarrow{\mathrm{q}} \cdot \overrightarrow{\mathrm{k}}+\frac{2}{\lambda} \mathrm{C}_{1}+\frac{2 \overrightarrow{\mathrm{s}} \cdot \overrightarrow{\mathrm{k}}-\lambda}{\lambda \mathrm{k}_{0}^{2}} \cdot \mathrm{C}_{2}\right) \\
& +\frac{F}{v_{1}^{2}\left(\Delta^{2}+1\right)}\left\{2|\vec{q}|^{2}|\vec{k}|^{2} \cdot \sin ^{2} \theta\right. \\
& \left.+\frac{2 c_{2}}{\lambda}\left[2(2 \vec{q}-\vec{k}) \cdot \vec{s}-\frac{(2 \vec{s} \cdot \vec{k}-\lambda)(2 \vec{q}-\vec{k}) \cdot \vec{k}}{k_{0}^{2}}\right]\right\} \\
& -\frac{F_{N}}{2 M \omega^{*} V_{1}^{2} V_{2}^{2}}\left\{|\vec{q}|^{2}|\vec{k}|^{2}\left(3-\cos ^{2} \theta\right)\right. \\
& \left.+\frac{4}{\lambda}\left[2(\vec{q} \cdot \vec{k} \times \vec{s})^{2}+c_{1} \vec{q} \cdot \vec{k}+c_{2} \vec{q} \cdot \vec{s}\right]\right\} \\
& -\frac{2\left(2 \vec{s} \cdot \vec{k}-|\vec{k}|^{2}\right)}{\lambda k_{0}^{2}}\left(F_{\pi}-F_{N}\right) \frac{C_{2}}{v_{I}^{2}}
\end{aligned}
$$

The following symbols were introduced:

$$
\begin{aligned}
& c_{1} \equiv(\vec{s} \times \vec{q}) \cdot(\vec{s} \times \vec{k}) \\
& c_{2} \equiv(\vec{k} \times \vec{q}) \cdot(\vec{k} \times \vec{s}) .
\end{aligned}
$$

The last term in Eq. (3.45) is the contribution of Eq. (3.18), the term that was added to $j_{B}$ to restore gauge invariance. The same result can be obtained by modifying the analysis following Eq. (3.4) to take account of a nonzero $k \cdot j$. 
A numerical calculation of these formulae as a function of $\theta$ has been made for the case discussed in Section II $C$, namely

$$
\begin{aligned}
& k_{p h}=500 \mathrm{Mev}, \quad \text { or } \quad E=9.657 \\
& T_{L}=5.039 \approx 700 \mathrm{Mev} .
\end{aligned}
$$

Two values of the angle $\varnothing$ (defined in Eq. (2.9)) were used: $\varnothing=0^{\circ}$ and $\varnothing=180^{\circ}$. From electron-nucleon scattering experiments the value of $F_{N}$ was chosen to be $F_{N V}(10)=0.62$.

To carry out the calculation it was necessary to estimate the value of the phase shift $\delta_{33}$ at $E=9.66$, which corresponds to pion-nucleon scattering at $350 \mathrm{Mev}$. One cannot use the Chew-Iow formula (3.4I) at such a high energy. At $307 \mathrm{Mev}$, Chiu and Lomon ${ }^{2 l}$ find a significant deviation. This is illustrated in Fig. 10, which shows the points of Chiu and Lomon and one point by Willis ${ }^{24}$ at $500 \mathrm{Mev}\left(\delta_{33}=157.3^{\circ}\right)$. In order to estimate the phase shift at $350 \mathrm{Mev}$, the points have been joined by a smooth curve and the value $\delta_{33}=145^{\circ}$ read off at $350 \mathrm{Mev}$. This crude estimate should be quite adequate for our purposes.

To make the dependence on the unknown form factor $F_{\pi}$ explicit, let us write

$$
\Phi=A(\theta, \varnothing) F_{\pi}^{2}+B(\theta, \varnothing) F_{\pi} F_{N}+c(\theta, \varnothing) F_{N}^{2} .
$$

The calculated dependence of $A, B$, and $C$ on $\theta$ is shown in Fig. Il for $\varnothing=180^{\circ}$ and in Fig. 12 for $\varnothing=0^{\circ}$. The physical meaning of the two values of $\varnothing$ is illustrated in FIg. 13. Comparison of the two cases reveals the fact that $A$ depends very strongly on $\varnothing$. The origin of 
this strong dependence is the term in brackets in the following expression for A:

$$
A=4\left(\frac{M}{E}\right)^{2} \frac{\Delta^{2}}{\lambda\left(\Delta^{2}+1\right)^{2}}\left[4(q \cdot s)\left(q \cdot s^{\prime}\right)-\lambda\right] .
$$

The term A is of course the pole term which has been discussed, but the extreme $\varnothing$ dependence noted above will be smoothed by the extrapolation. procedure suggested in Section II B. Recall that it is necessary to symmetrize the cross section as prescribed by Eq. (2.7) in order to eliminate a branch point. The symmetrized $\Phi$ can equivalently be defined as

$$
\varnothing_{s}(\theta, \varnothing) \equiv \Phi(\theta, \varnothing)+\Phi(\theta, \varnothing+\pi)
$$

This symmetrized $\varnothing_{S}$ is then the: average of the $\varnothing=\pi$ case of.Fig. 11 and the $\varnothing=0$ case of Fig. 12 .

Before going on to the consideration of $\varnothing_{S}$ and its extrapolation, let us note the dependence of the electroproduction cross section on the parameter $F_{\pi}(10)$, with $\left.F_{N N}(10)=0.62\right)$. Several cases are plotted in Fig. 14. One interesting feature of this graph is that for any curve $F_{\pi}(10)=\beta$, where $\beta \leqslant 0.85$, there is another very similar curve corresponding to a different value of $F_{\pi}(10)$. This is illustrated in Fig. 14 by the curves for $F_{\pi}(10)=F_{N}(10)=0.62$ and for $F_{\pi}=0.20$. Finally, Fig. 15 shows the calculated behavior of the extrapolation function $\left(\Delta^{2}+1\right)^{2} \varnothing_{s}$ at the end of the physical region. 


\section{CONCLUSIONS}

We are now able to apply the error estimates developed in Section II C. For example, recall that for one case in which the cross section was measured at nine specific points, the error was found to be $\Delta_{0}^{-} \approx 100 \xi$. If $\Delta_{0}$ is the error in $\left(\Delta^{2}+1\right)^{2} \Phi_{s}$ at the point $\Delta^{2}=\infty 1$, then $\xi$ is the error (assumed constant) in $\Phi_{s}$ at each measured point. From Fig. 15 we see that if $\Delta a_{0} \gtrsim 2$ the extrapolation yields no useful information. This requires a constant absolute error in $\varnothing_{s}$ less than 0.02. Fig. 14 shows that if $F_{\pi}=0.62$, this means a percent error ranging from $2 \%$ at $\theta=0$ to $6 \%$ at $\theta=90^{\circ}$ and $20 \%$ at $\theta=180^{\circ}$. Even this estimated upper limit of accuracy is considerably better than that achieved by Panofsky and Allton ${ }^{2}$ in an electroproduction experiment in which only $E$ and $\lambda$ (not $\Delta^{2}$ ) were determined. Thus it appears that the proposed extrapolation, although possible in principle, will require experiments of great accuracy. 


\section{ACKINOWLEDGMENTS}

The problem considered in this thesis was suggested by Professor Geoffrey F. Chew, whose valuable advice throughout the course of this work I greatly appreciate. The analysis of error in a least-squares extrapolation was done with the help of Dr. Michael J. Moravcsik. I also benefited greatly from very frequent discussions with James S. Ball and Peter Cziffra.

This work was performed under the auspices of the United States Atomic Energy Commission. 


\section{FIGURE LEGENDS}

Fig. 1. Electropion production, considered in lowest order in $\alpha$. Single lines are èlectrons; double lines, nucleons; wavy lines, photons; and broken lines, pions.

Fig. 2. Poles and branch points in the $\cos \theta$ plane. The scale of the figure is appropriate to the values $E=9.66, \lambda=10$. The rightrhand pole is the one at $\Delta^{2}=-\mu^{2}$.

Fig. 3. The class of diagrams giving rise to the pole at $\Delta^{2}=-\mu^{2}$. Fig. 4. The pion-photon vertex.

Fig. 5. The distance of extrapolation as a function of $E$ for two values of $\lambda_{0}$ Also shown in $k_{p h}$, the equivalent laboratory energy in photoproduction $\left(E^{2}=M^{2}+2 M k_{p h}\right)$ 。

Fig. 5a. The dependence of the extrapolation error $\Delta a / \xi$ on $E$.

Fig. 6. The variation of the symmetrized residue at the pole as a function of $E$ for various $\varnothing$ at $T_{L}=700 \mathrm{Mev}$ and $\lambda=10$.

Fig. 7. $\mathrm{E}^{2}$. vs. $\lambda$ for $\mathrm{T}_{\mathrm{L}}=700 \mathrm{Mev}$ for various values of the laboratory scattering angle $\psi$ of the final electron. Points above the line $\psi=\pi$ are not kinematically possible。

Fig. 8. The diagrams giving rise to the nucleon-current Born terms in the electroproduction dispersion relations.

Fig. 9. Photoproduction at $400 \mathrm{Mev}$. Solid line: prediction of modified CGLN formula, Eq. (3.40). Dashed line: prediction of CGIN formula. Experimental points are those of Walker et al. 23 
Fig. 10. The phase shift $\delta_{33^{\circ}}$. Point at $500 \mathrm{Mev}$ by Willis; ${ }^{24}$ others by Chiu and Lomon. 21 The dotted curve has been drawn to estimate $\delta_{33}$ at $350 \mathrm{Mev}$. The solid line is the continuation of the Chew Low fit to the low-energy region, with $f^{2}=0.08$ and $\omega_{r}=2.1$.

Fig. 11. The dimensionless functions $A(\theta), B(\theta)$, and $C(\theta)$ defined by

$$
\Phi=A F_{\pi}^{2}+B F_{\pi} F_{N}+C F_{N}^{2} \text {, }
$$

for $\varnothing=180^{\circ}$.

Fig. 12. The functions $A(\theta), B(\theta)$; and $C(\theta)$ for $\varnothing=0^{9}$.

Fig. 13. Illustration of the two cases $\varnothing=180^{\circ}$ and $\varnothing=0^{\circ}$ in terms of momenta in the system in which $\vec{p}^{8}+\vec{q}=0$.

Fig. 14. A plot of the dimensionless function $\Phi$, proportional to the electroproduction cross section, for $\mathrm{E}=9.66, \lambda=10$, $\mathrm{T}_{\mathrm{L}}=700 \mathrm{Mev}, \varnothing=\pi$, and various values of the pion form factor.

Fig. 15. The calculated behavior of the extrapolation function $\left(\Delta^{2}+1\right)^{2} \Phi_{s}$ at the end of the physical region for the case discussed. 
REF'ERENTES

1. Hofstader, Bumillex, and Yerian, Revs. Modern Phys. 30, 482 (1958).

2. W. K. H. Panofsky and E. A. Aliton, Phys. Rev. 110, 1155 (1958).

3. Chew, Karplus, Gasiorowicz, and Zachariasen, Phys. Rev。 110, 265 (1958).

4. Federbush, Goldberger, and Treiman, Phys.Rev. 112, 642 (1958).

5. G. F. Chew and F.E. Low, Unstable Particles as Trargets in Scattering Experiments, UCRL-8427, August 21, 1958.

6. Geoffrey F. Chew, Phys. Rev. 112, 1380 (1959).

7. Po. Cziffre and M。 J. Moravcsik, Bull. Am. Phys. Soco, Seroies II, 2. 404. (1958)。

8. Taylor, Moravesik, and Uretsky, Determination of the Pion-Nucleon Coupling Constant from Photoproduction Angular Distribution, UCRL 8442 , September 10, 1958; quoted in the following as TMU.

9. Chew, Goldberger, Low, and Ilambu, Phys. Rev. 106, 1345 (1957); quoted in the following as CGINT. See also Geoffrey Fo Chew, Theory or Pion Scattering and Photoproduction, Encyelopedia of Physics, 2nd ed., Vol. 43 (Spxinger-Verlag, Berlin, to be published).

10. Fubini, Nambu, and Wataghin, Phys. Rev。 111, 329 (1958); quoted in the following as FWW.

11. S. Mandelstam, Phys。Rev. 112, 1344 (1959)。

12. P. Caiffra and M. J. Moravcsik; A Practlcal Guide to the Method of Least Squares, UCRL-8523, Oçtober 17, 1958.

13. R. H. Dalitz and D. R. Yennie, Phys. Rev. 105, 1598 (1957); quoted in the following as DY.

14. McDonald, Peterson, and Corson, Phys. Rev。 107, 577 (1957)。 
15. Uretsky, Kenney, Knapp, and Perez-Mendez, Phys. Rev. Letters 1, 12 (1958)。

16. Iazarus, Panofsky, and Tangherlini, Phys. Rev., to be published.

17. R. Oehme and J. G. Taylor, Phys. Rev,, to be published.

18. See, for example, Yennie, Levy, and Ravenhall, Revs. Modern Phys.29, 144 (1957).

19. Wolfgang K. H. Panofsky, in Proceedings of the 1958 Annual Internationai Conference on High Energy Physics at CERN (CERN; Geneva). See especially p. 18 .

20. For a readable explanation of the analysis of photoproduction into multipole amplitudes, see M. J. Moravcsik, Selected Topics in Low Energy Pion Physics, Office of Technical Services, Department of Commerce, Washington 25, D。C., 1958. See especlally pp. 15-16.

21. H. Y. Chiu and E. I. Lomon, Annals of Physies 6, 50 (1959).

22. G. Puppi, in Proceedings of the 1958 Annual International Conference on High Energy Physics at CERN (CERN, Geneva), p. 42.

23. Walker, Teasdale, Peterson, and Vette, Phys. Rev. 99, 210 (1955).

24. Reported by Oreste Piccioni in Proceedings of the 1958 Annual International Conference on High Energy Physics at CERN (CERN, Geneva), p. 67 . 
$-50-$

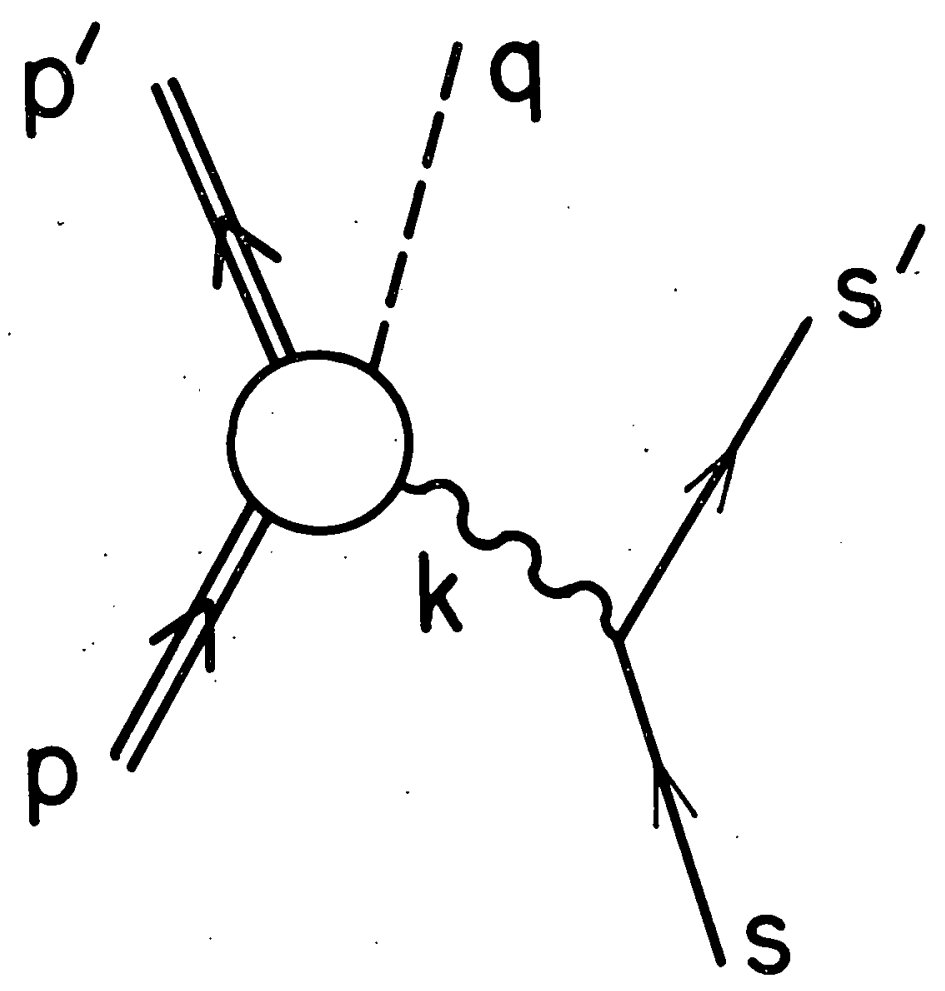

MU - 16922

Fig. 1 . 


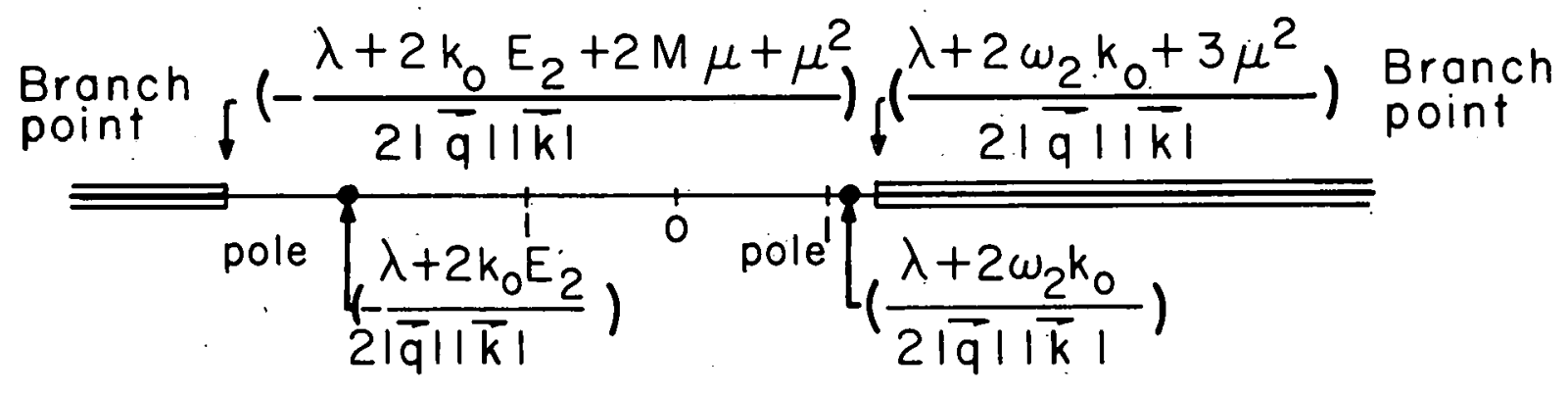

$M U-16914$

Fig. 2 . 
$-52-$

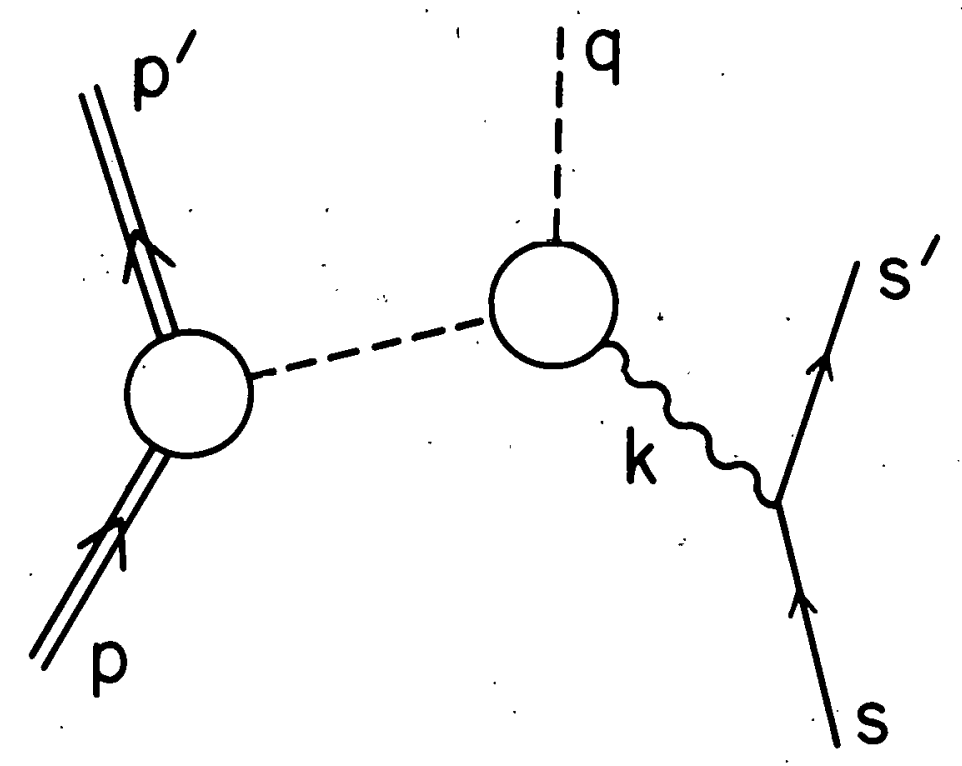

$M U-16921$

Fig. 3 . 
$-53-$

$q_{2} !$

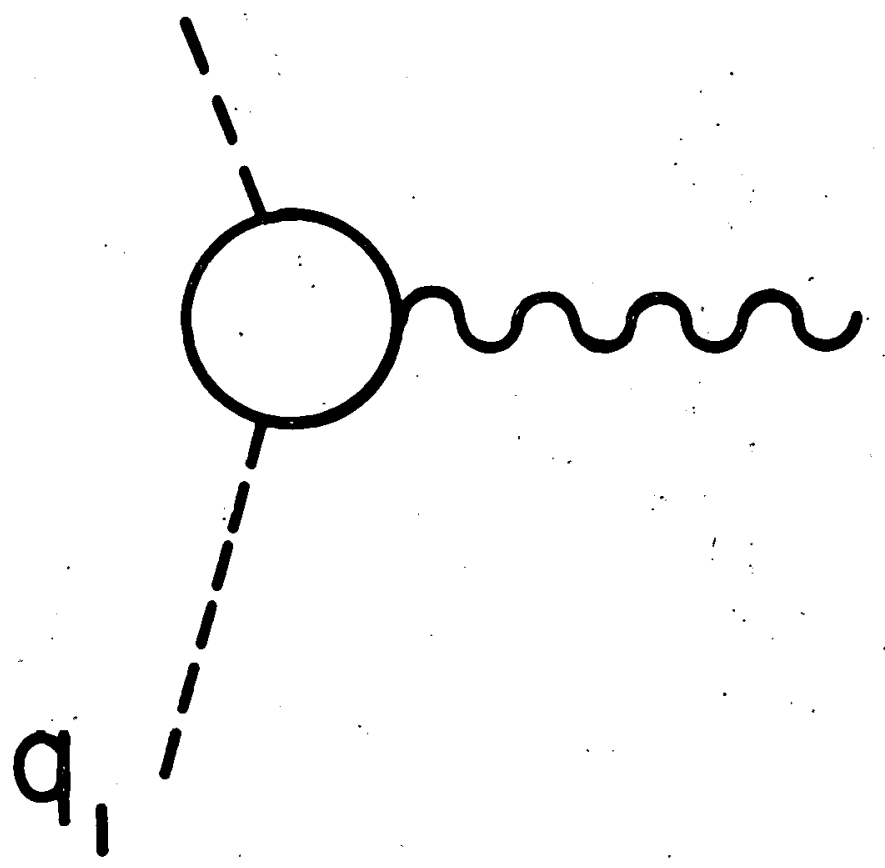

$M U-16925$

Fig. 4. 


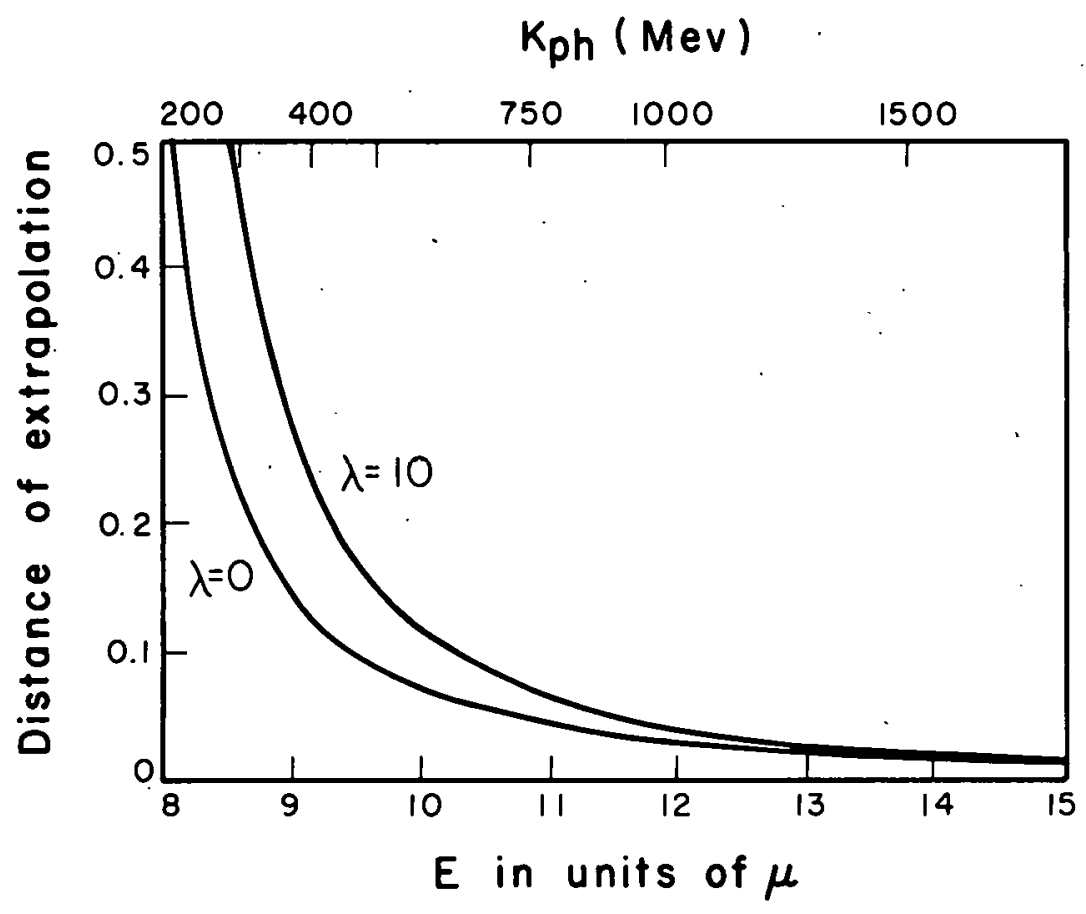

$M U-16919$

Fig. 5 


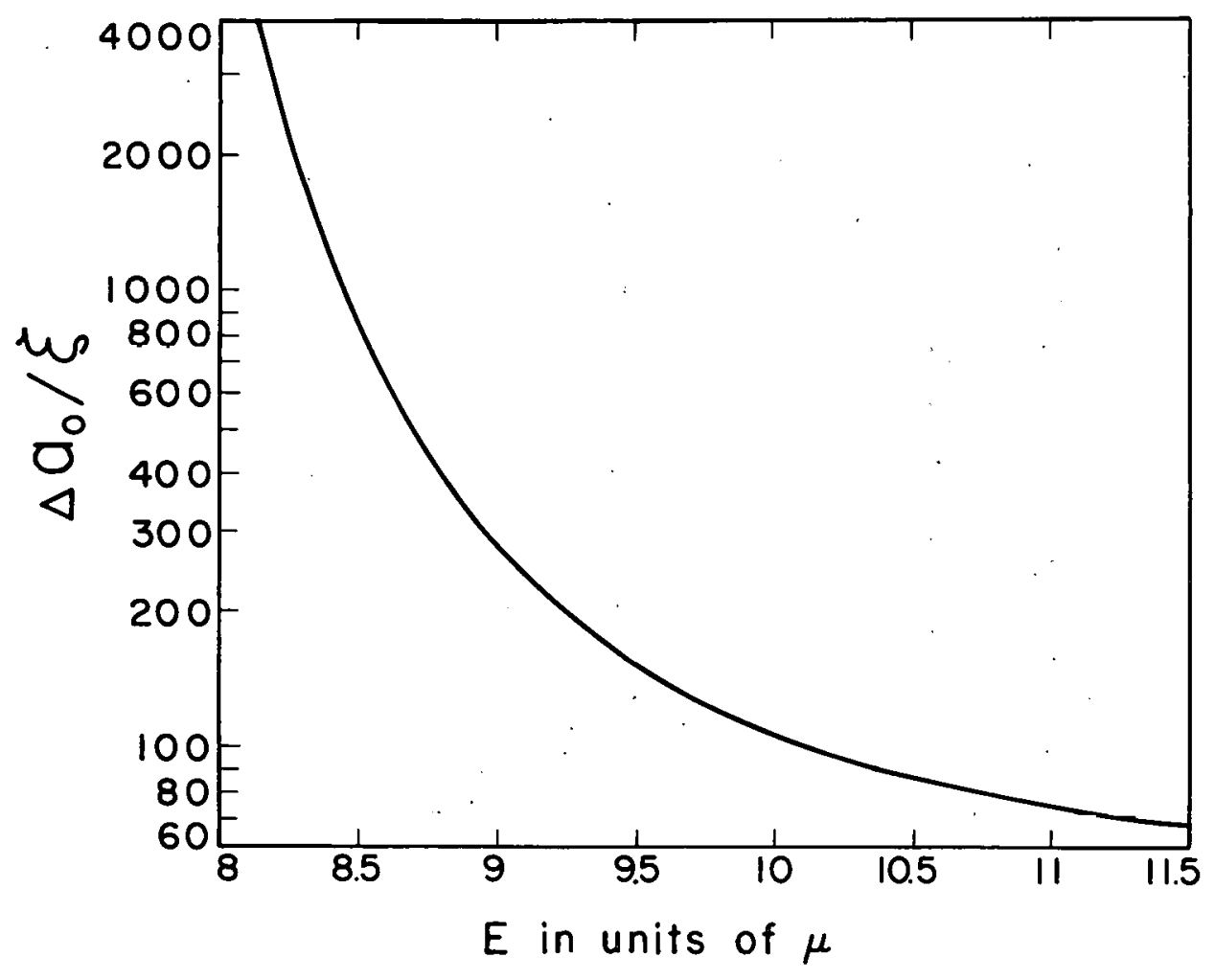

$M U-16917$

Fig. 5 a 


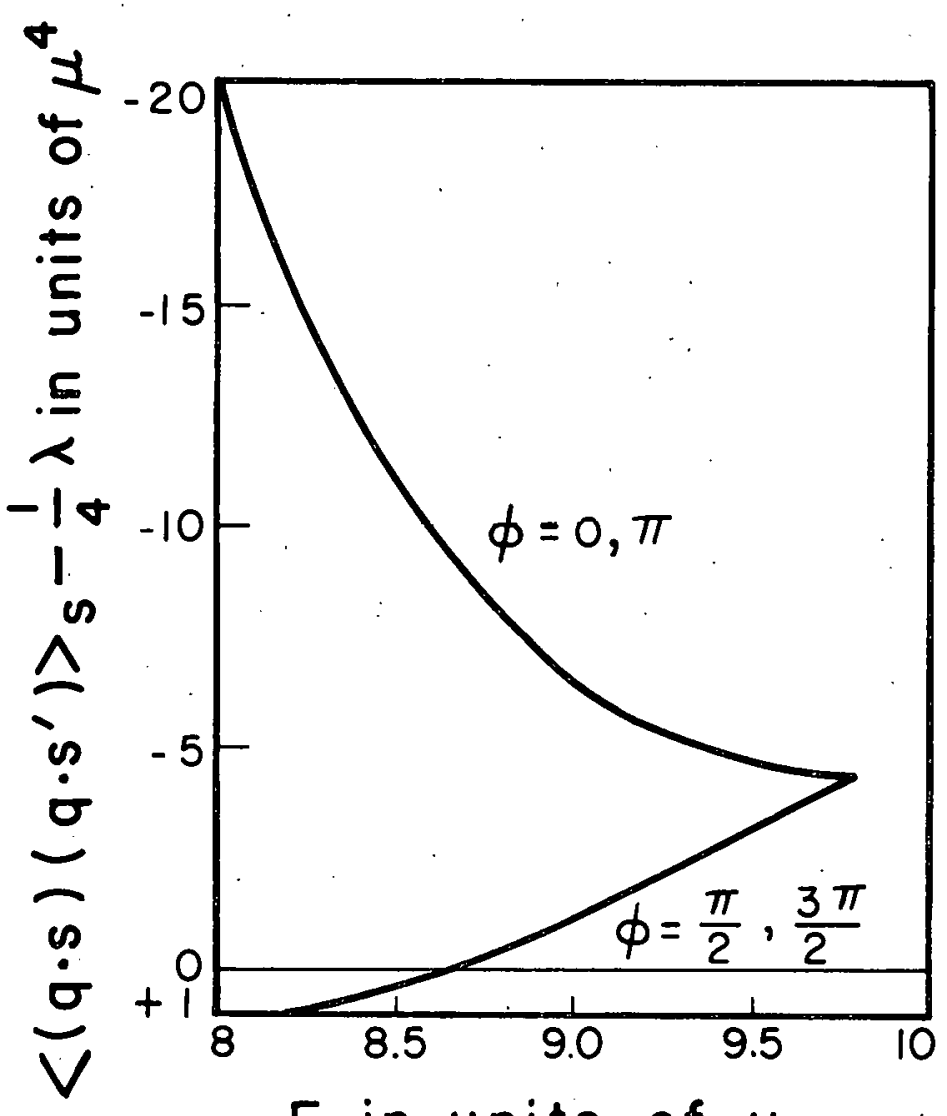

$E$ in units of $\mu$

$$
M U-16926
$$

Fig. 6. 


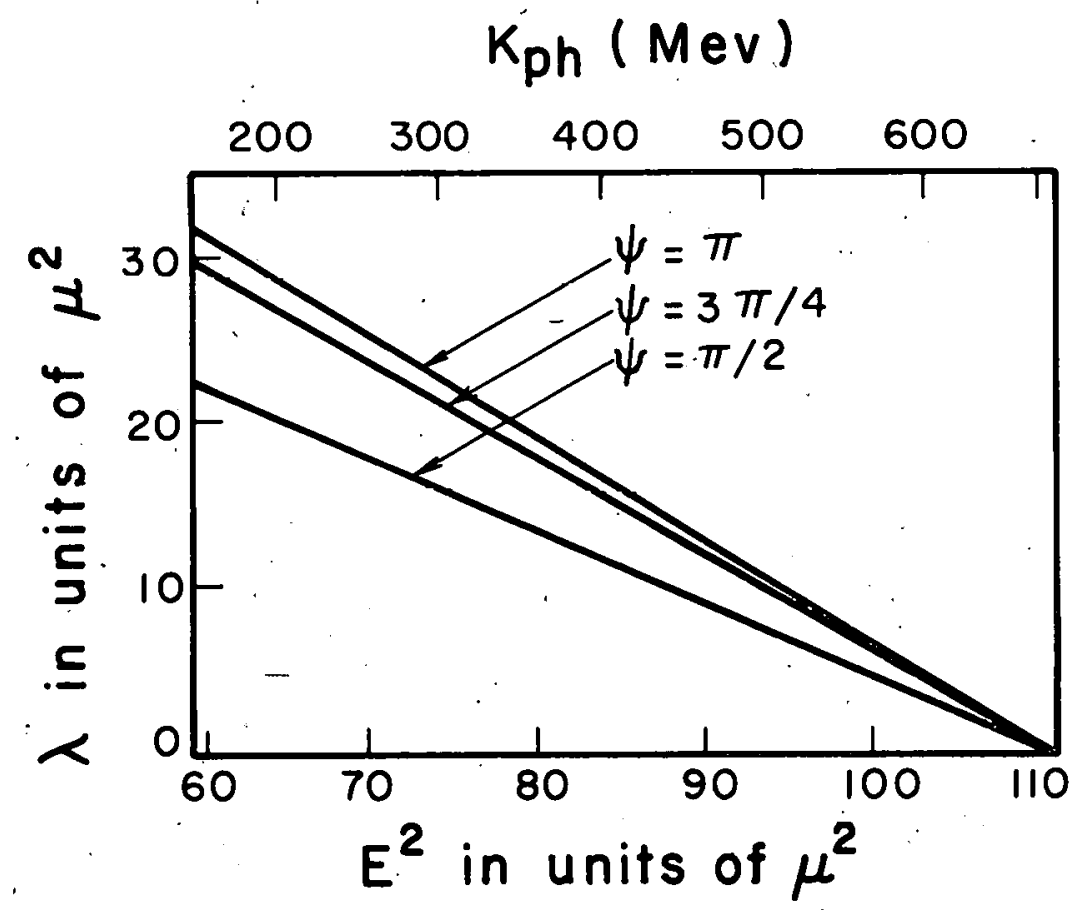

$M U-16918$

Fig. 7 . 
$-58-$
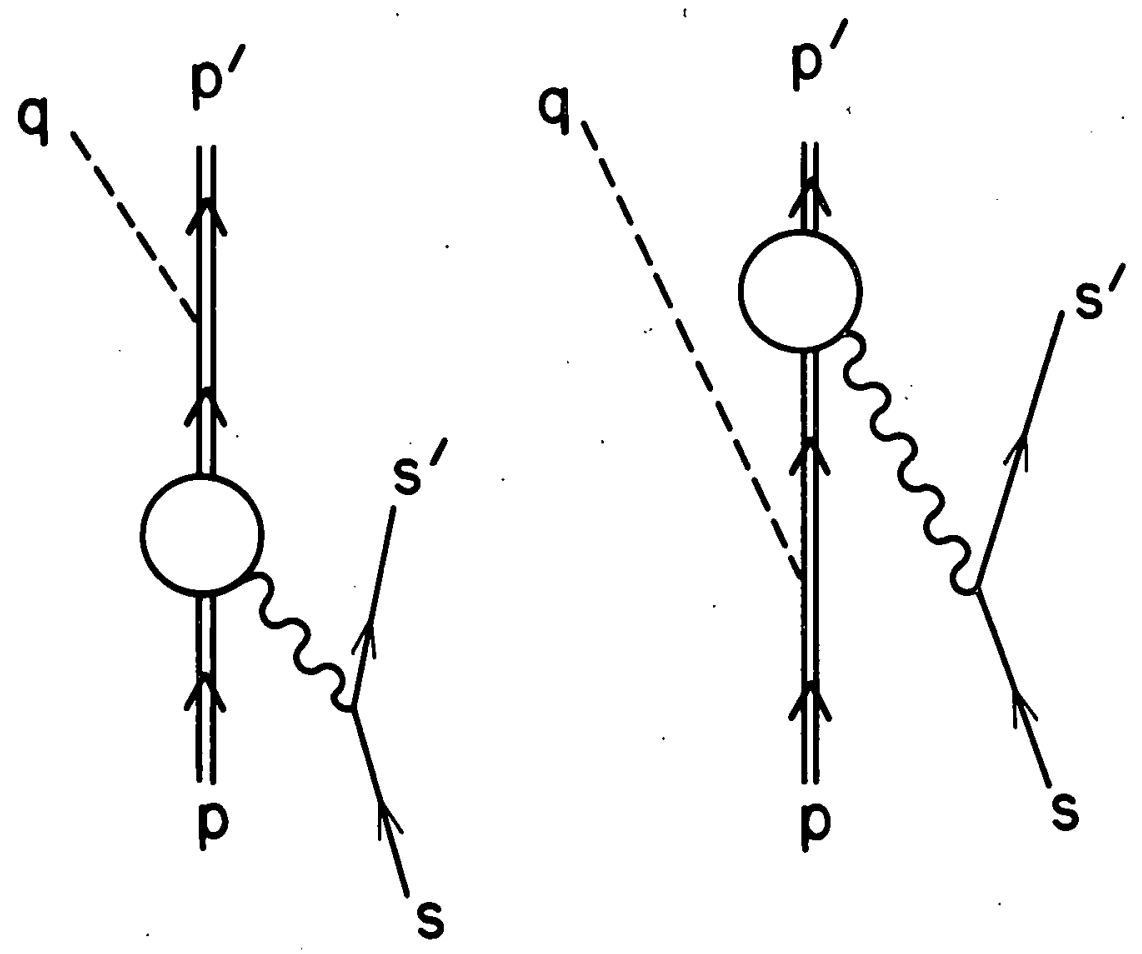

$M U-16924$

Fig. 8. 


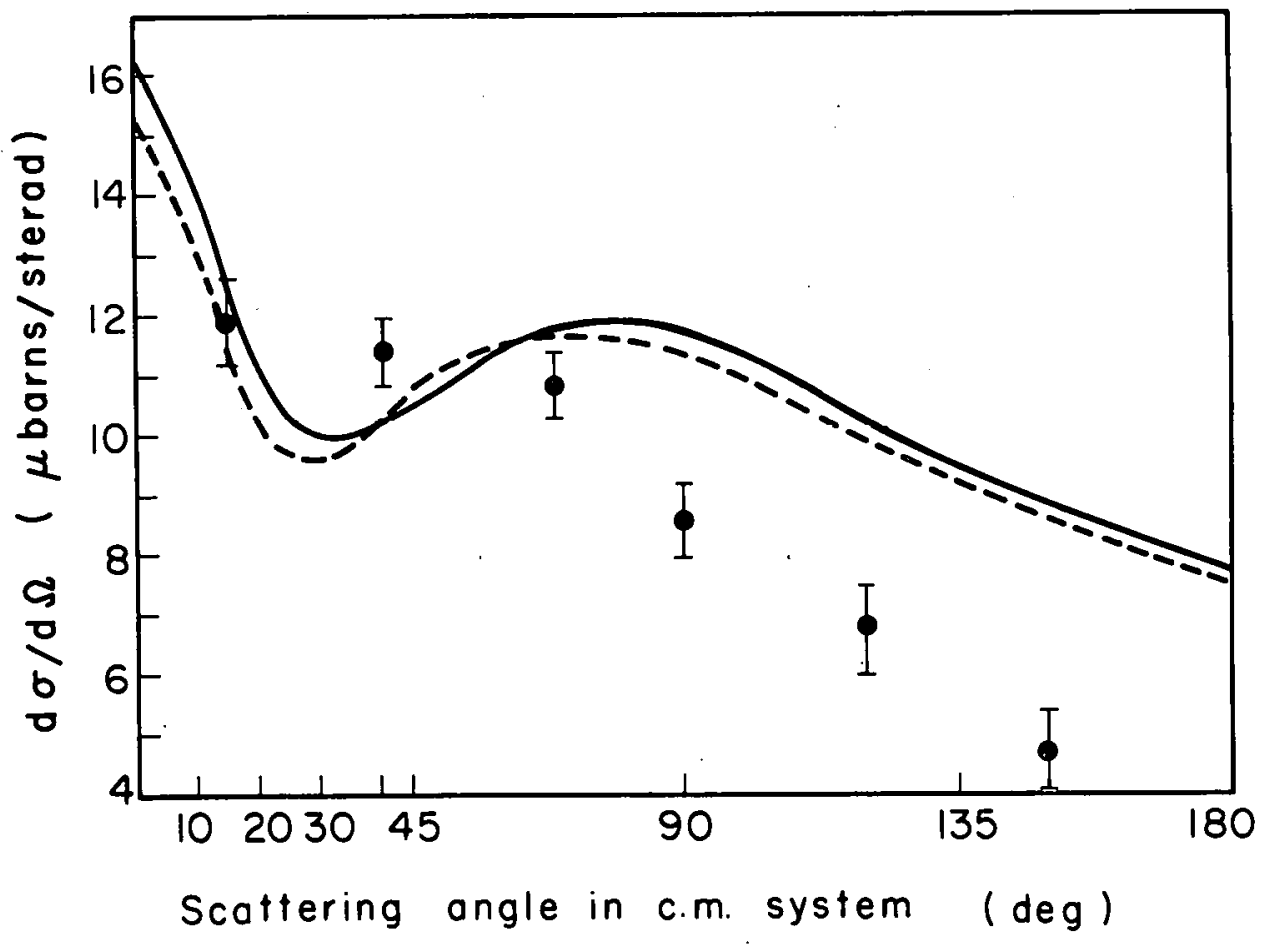

$M U-16920$

Fig. 9. 


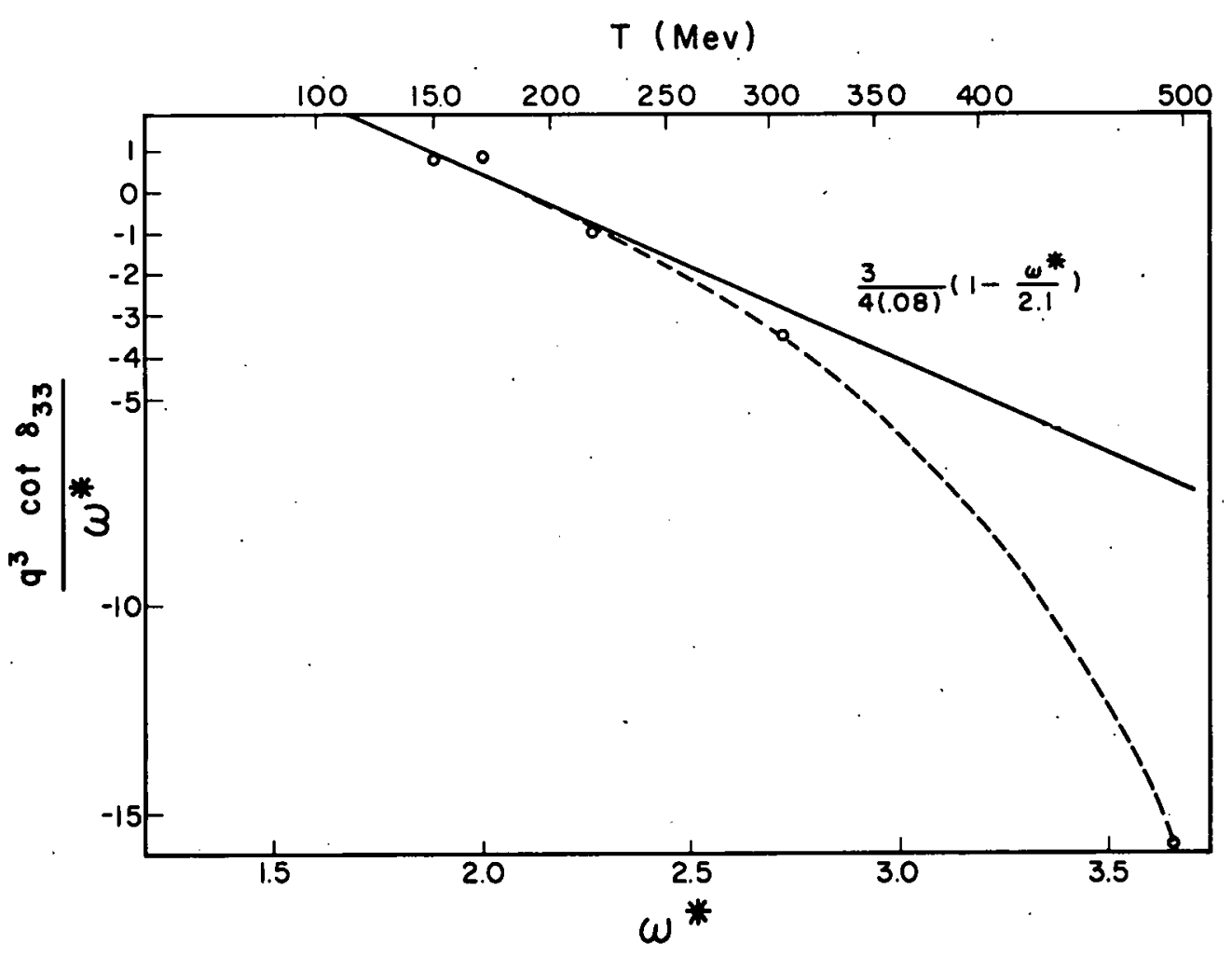

$M U-16915$

Fig. 10 . 


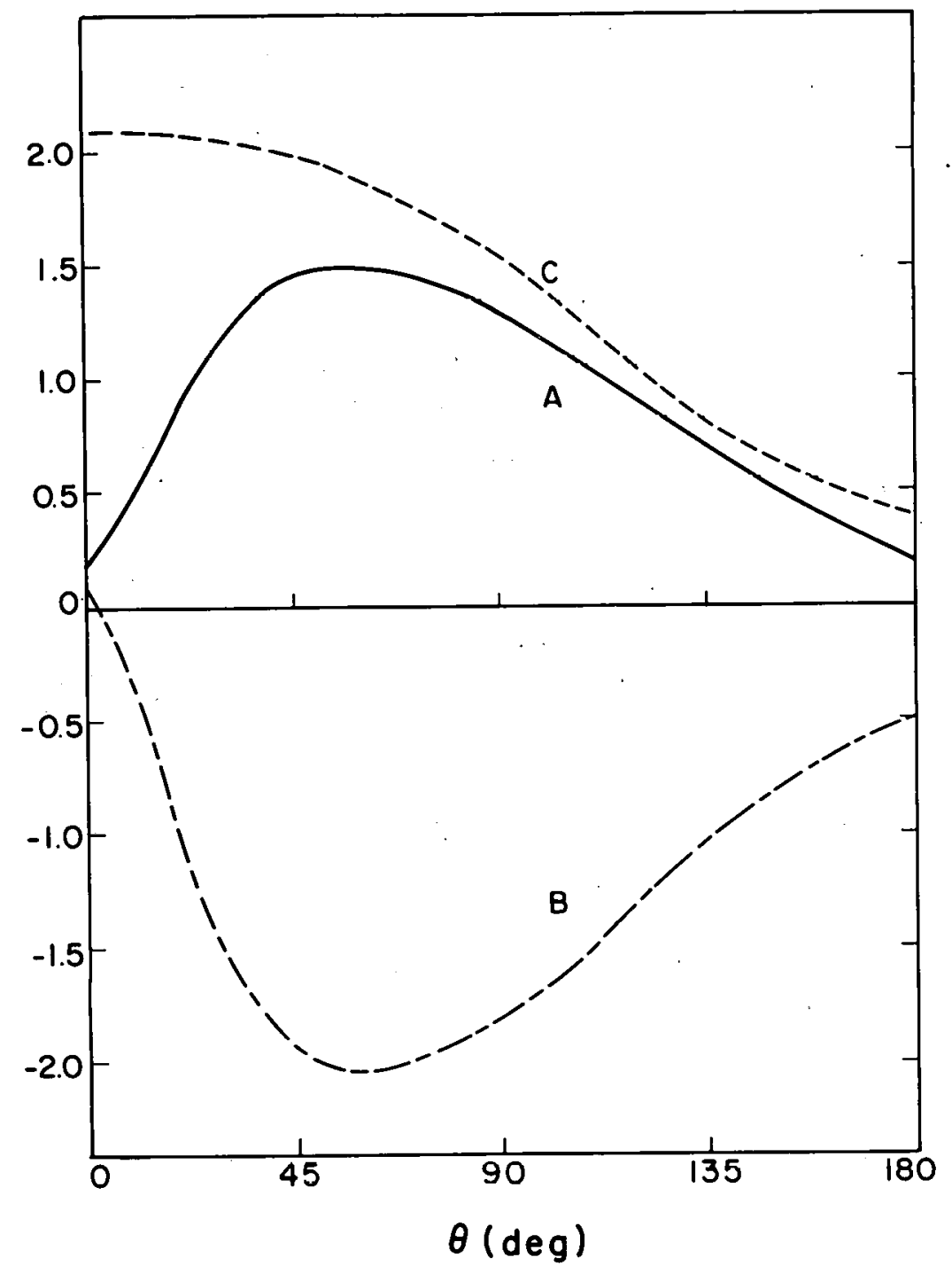

$M U-16927$

Fig. 11. 
$-62-$

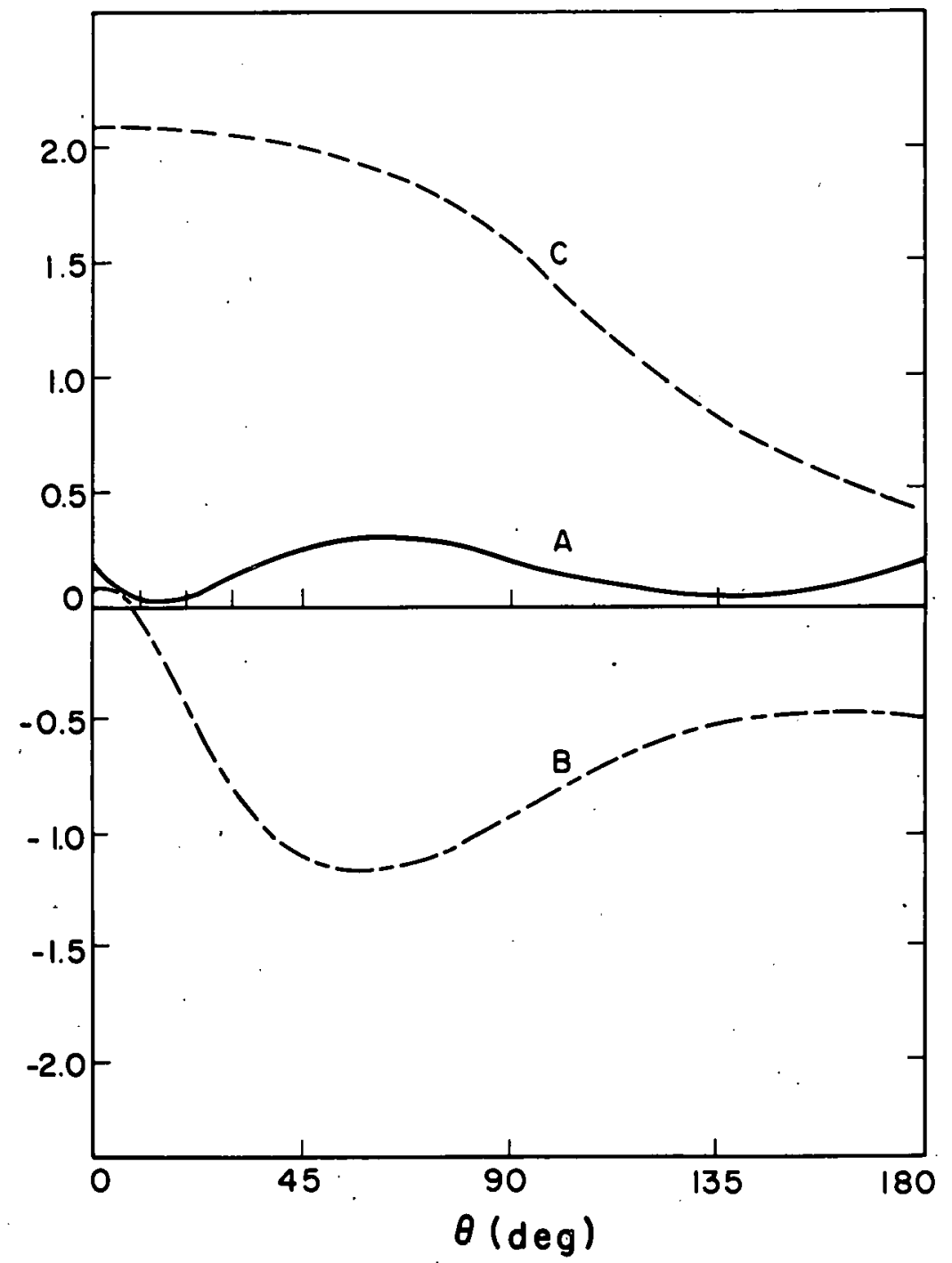

$M U-1.6928$

Fig. 12 

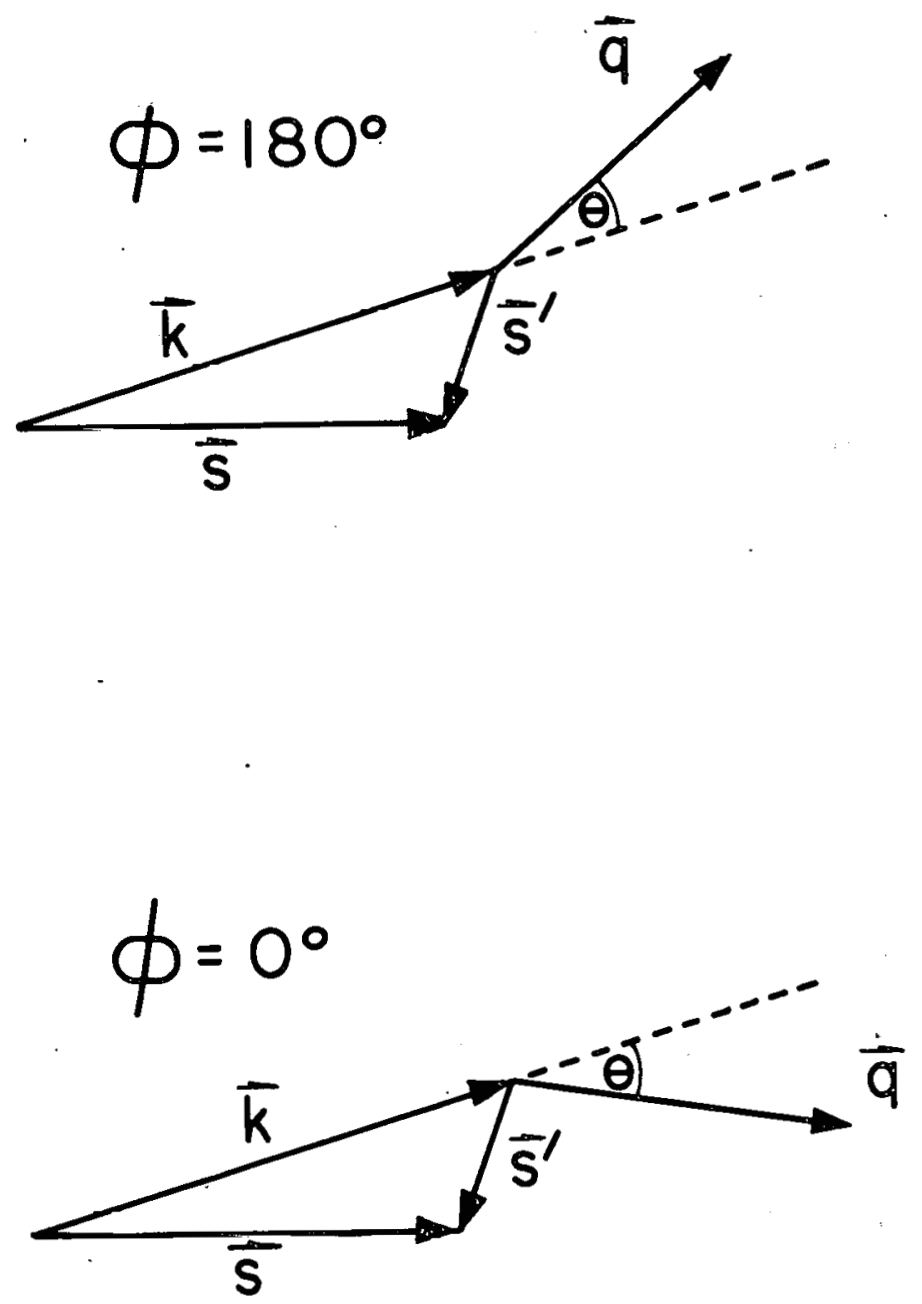

MU - 16923

Fig. 13 


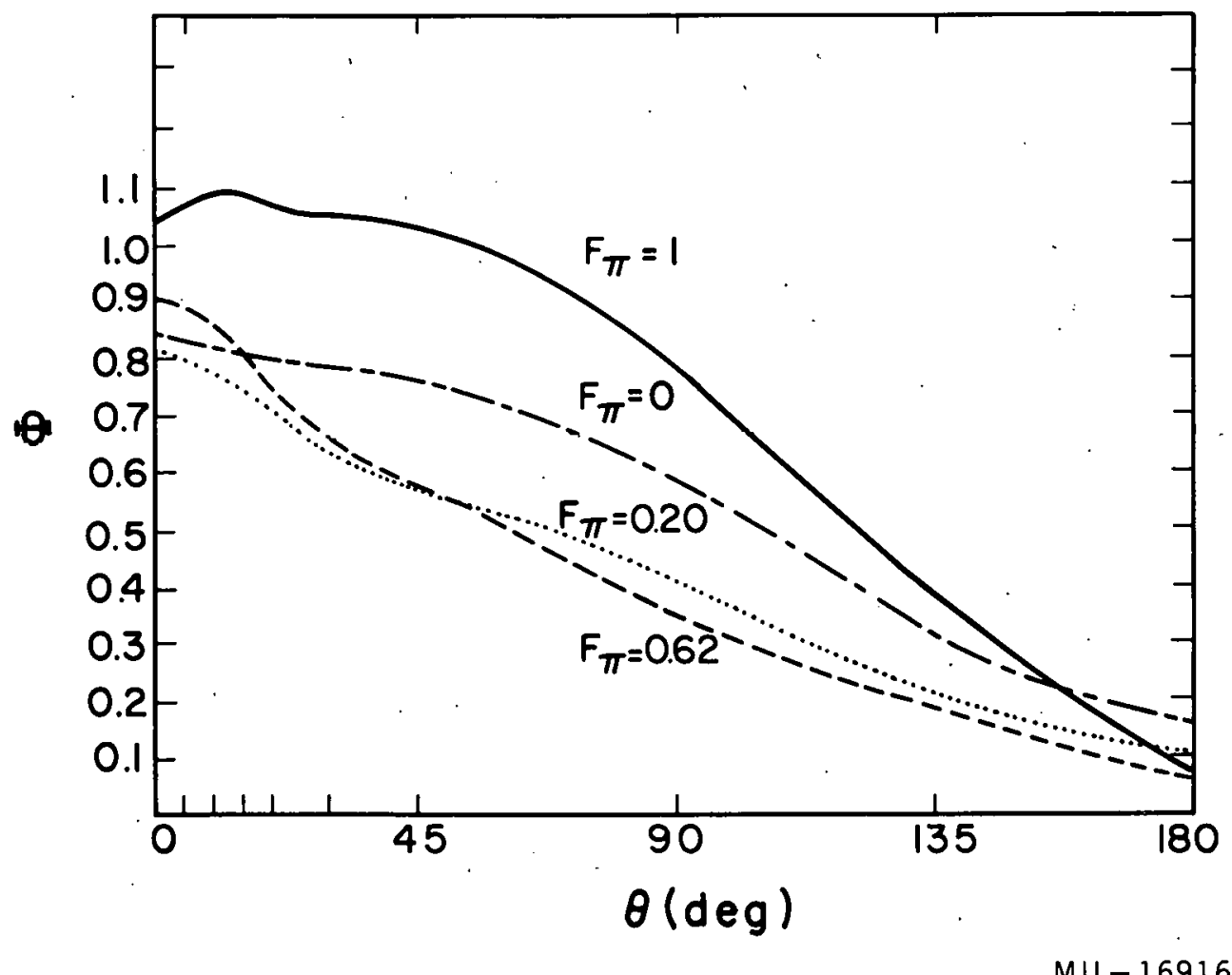

Fig. 14 
$-65-$

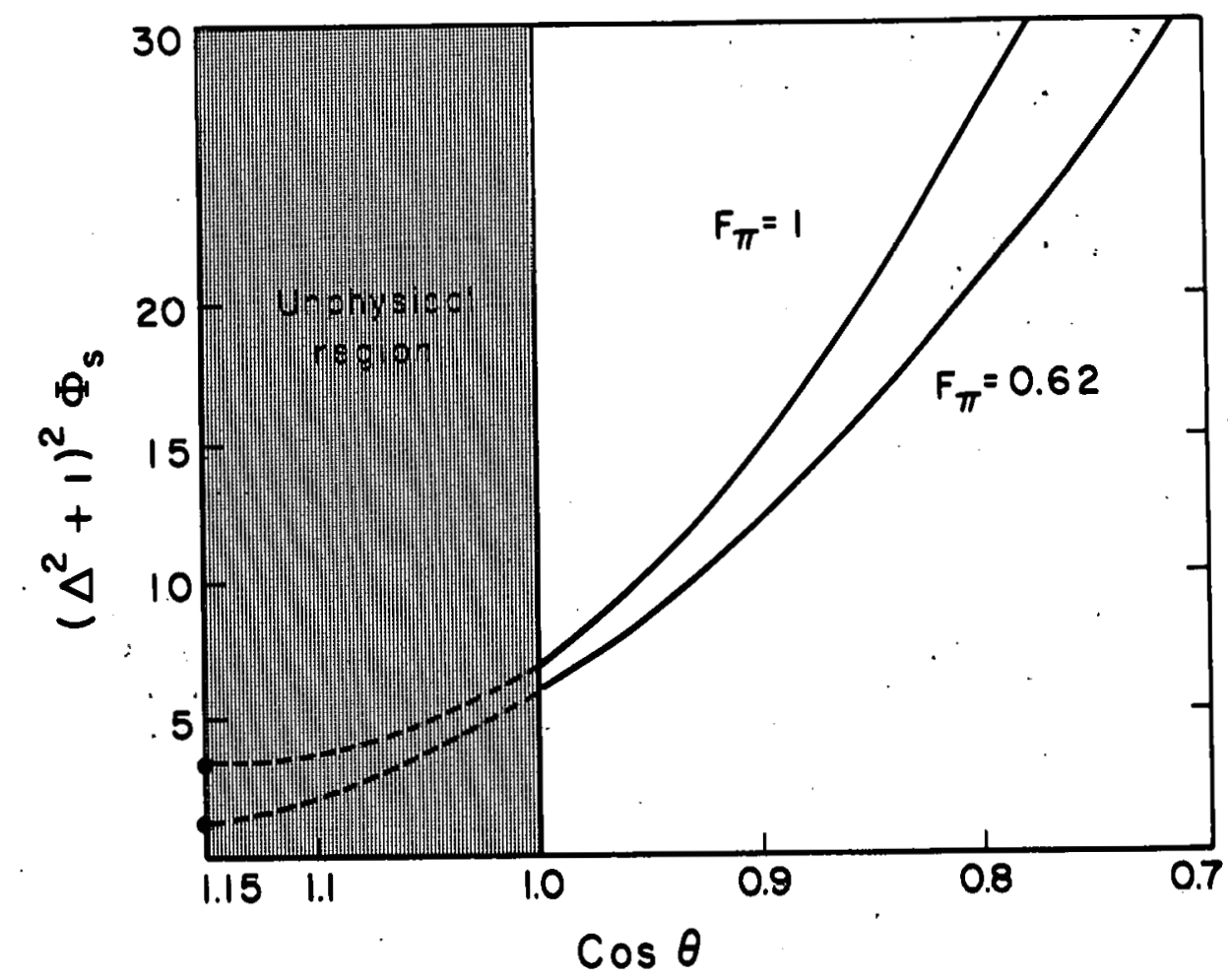

Fig. 15. 
This report was prepared as an account of Government sponsored work. Neither the United States, nor the Commission, nor any person acting on behalf of the Commission:

A. Makes any warranty or representation, expressed or implied, with respect to the accuracy, completeness, or usefulness of the information contained in this report, or that the use of any information, apparatus, method, or process disclosed in this report may not infringe privately owned rights; or

B. Assumes any liabilities with respect to the use of, or for damages resulting from the use of any information, apparatus, method, or process disclosed in this report.

As used in the above, "person acting on behalf of the Commission" includes any employee or contractor of the Commission, or employee of such contractor, to the extent that such employee or contractor of the Commission, or employee of such contractor prepares, disseminates, or provides access to, any information pursuant to his employment or contract with the Commission, or his employment with such contractor. 